\title{
Noninvertible Gabor Transforms
}

\author{
Ewa Matusiak, Tomer Michaeli, Student Member, IEEE, and Yonina C. Eldar, Senior Member, IEEE
}

\begin{abstract}
Time-frequency analysis, such as the Gabor transform, plays an important role in many signal processing applications. The redundancy of such representations is often directly related to the computational load of any algorithm operating in the transform domain. To reduce complexity, it may be desirable to increase the time and frequency sampling intervals beyond the point where the transform is invertible, at the cost of an inevitable recovery error. In this paper we initiate the study of recovery procedures for noninvertible Gabor representations. We propose using fixed analysis and synthesis windows, chosen e.g., according to implementation constraints, and to process the Gabor coefficients prior to synthesis in order to shape the reconstructed signal. We develop three methods for signal recovery. The first follows from the consistency requirement, namely that the recovered signal has the same Gabor representation as the input signal. The second, is based on minimization of a worst-case error. Last, we develop a recovery technique based on the assumption that the input signal lies in some subspace of $L^{2}$. We show that for each of the criteria, the manipulation of the transform coefficients amounts to a 2D twisted convolution, which we show how to perform using a filter-bank. When the undersampling factor is an integer, the processing reduces to standard $2 D$ convolution. We provide simulation results demonstrating the advantages and weaknesses of each of the algorithms.
\end{abstract}

Index Terms- Gabor transform, sampling, twisted convolution.

\section{INTRODUCTION}

$\mathbf{T}$ IME-FREQUENCY analysis has become a popular tool in signal processing. During the past three decades, it has been successfully used for noise suppression [1], [2], blind source separation [3], echo cancellation [4], [5], relative transfer function identification [6], beamforming in reverberant environments [7], system identification in general [8], and more. In algorithms based on time-frequency transforms such as the Gabor representation, there is often a tradeoff between performance and computational complexity, which can be controlled by adjusting the redundancy of the transform. The latter is determined by the product of the sampling intervals in time and frequency, which we denote by $a$ and $b$, respectively. Specifically, as $a$ and $b$ are increased, there are less coefficients

Manuscript received July 01, 2009; accepted January 07, 2010. Date of publication February 05, 2010; date of current version April 14, 2010. The associate editor coordinating the review of this manuscript and approving it for publication was Dr. Peter J. Schreier. The work of E. Matusiak was supported in part by Technion by a fellowship of the Israel Council for Higher Education. This work was supported in part by the Israel Science Foundation by Grant 1081/07 and by the European Commission in the framework of the FP7 Network of Excellence in Wireless COMmunications NEWCOM++ (contract 216715).

The authors are with the Department of Electrical Engineering, Technion-Israel Institute of Technology, Haifa, Israel (e-mail: ewa.matusiak@univie.ac.at; tomermic@tx.technion.ac.il; yonina@ee.technion.ac.il).

Color versions of one or more of the figures in this paper are available online at http://ieeexplore.ieee.org.

Digital Object Identifier 10.1109/TSP.2010.2042480 per time unit for any given frequency range, and therefore the amount of computation needed to process them decreases.

An important example in which the performance-complexity tradeoff is controlled by the redundancy of the transform, is that of system identification in the time-frequency domain [8]. In this setting the LTI system to be identified is often modeled by a sub-band filtering structure, in which each frequency bin is convolved with a finite-impulse response (FIR) filter. The goal is to identify these sub-band filters given noisy observations of the input and output of the system. It is clear that the smaller $a$ and $b$, the more data that has to be processed. On the other hand, as the redundancy is increased, a model mismatch error is introduced, since the sub-band filtering structure seizes to constitute a loyal model for the LTI system. This tradeoff was thoroughly investigated in [8].

The signal processing literature on Gabor-domain algorithms heavily relies on the fundamental requirement that any signal can be recovered from its coefficients in the transform domain. This requirement leads to the upper bound $a b \leq 1$. However, since the performance-complexity tradeoff is of continuous nature, it seems very restrictive to limit the discussion to this regime. Specifically, by increasing the sampling intervals beyond this bound we may further reduce the computational load of any algorithm operating in the Gabor domain. This benefit is obtained at the expense of deterioration in performance. It is important to note that when $a b>1$, an additional source of performance degradation comes into play, which is the inherent reconstruction error. This is because, as we show, in this regime, we can only guarantee perfect reconstruction for signals lying in certain subspaces of $L^{2}$, but not for the entire space. Nevertheless, the resulting complexity reduction may be of greater value in some applications.

In this paper, we explore reconstruction techniques for noninvertible Gabor transforms, namely in which $a b>1$. The fact that in these cases perfect recovery cannot be guaranteed for every signal introduces extra flexibility in choosing the analysis and synthesis windows of the transform. Specifically, we address the case where both the analysis and synthesis windows are specified in advance. They can be chosen according to implementation considerations, for example finite support windows, or certain multiple-pole windows [9] admitting an efficient recursive implementation. Our goal, then, is to process the transform coefficients before reconstruction such that the recovered signal possesses certain desired properties.

To treat this problem, we borrow several approaches from the field of sampling theory, which has reached a high degree of maturity in recent years [10], [11]. We begin by employing the consistency criterion in which the recovered signal $\tilde{f}(t)$ is constructed such that its Gabor transform coincides with that of $f(t)$ [12]. We then analyze a minimax strategy, where the reconstruction error $\|\tilde{f}-f\|$ is minimized for the worst-case 
input $f(t)$ [13]. Both these approaches are prior-free in the sense that they do not make use of any special properties, or prior knowledge, that might be available on the signal.

A prevalent prior in the sampling literature, is that the signal to be recovered lies in a shift invariant (SI) subspace of $L^{2}$ (see, e.g., [14]-[18] and references therein). In fact, signals and images encountered in many applications can be quite accurately modeled as belonging to some SI space [10], [11], such as the space of bandlimited functions, the space of polynomial splines and more. Their widespread use can also be attributed to the link that subspace priors have with stationary stochastic processes [19]-[22], which have been shown to constitute realistic priors for the behavior of natural images [23]. In this paper, we generalize the SI-prior used in the sampling community to a richer type of subspaces of $L^{2}$, which we term shift-and-modulation (SMI) invariant spaces (see also [24]). The third class of inverse Gabor techniques we consider, then, makes use of the SMI prior. We show that such a prior can lead to perfect recovery in some cases, given that the synthesis window is chosen according to the prior. For a fixed synthesis window, which is not matched to the prior, we show how to achieve the minimal possible reconstruction error for signals in the prior-space.

In each of the three techniques we develop, the processing of the Gabor coefficients amounts to a 2D twisted-convolution [25] with a certain kernel, which depends on the analysis and synthesis windows. We show that the twisted-convolution operation can be interpreted in terms of a filter-bank. Furthermore, in the case of integer undersampling (i.e., when $a b$ is an integer), the resulting process reduces to a standard $2 \mathrm{D}$ convolution in the timefrequency domain. In these cases, we discuss situations in which the $2 \mathrm{D}$ convolution kernel is a separable function of time and frequency. This allows a significant reduction in computation, namely by implementing the $2 \mathrm{D}$ convolution as two successive 1D filtering operations along the time and frequency directions.

The paper is organized as follows. Section II is devoted to notation that will be used throughout the paper. In Section III we derive conditions on the analysis and synthesis windows such that they generate Riesz bases for their span, which guarantees that the noninvertible Gabor representation is stable. In Section IV we review sampling and reconstruction schemes in SI spaces in order later to be able to generalize them to the Gabor transform using SMI spaces. Sections V, VI, and VII constitute the central part of the paper, where in the first two we develop prior-free recovery procedures for Gabor transforms in the integer and rational undersampling regimes, respectively, and in the last we discuss SMI-prior recoveries. Finally, in Section VIII we demonstrate the methods we develop for the case in which both the analysis and synthesis are performed with Gaussian windows.

\section{NOTATION AND DEFINITIONS}

We will be working throughout the paper with the Hilbert space of complex square integrable functions, denoted by $L^{2}(\mathbb{R})$, with inner product

$$
\langle f, g\rangle=\int_{-\infty}^{\infty} f(t) \overline{g(t)} d t \quad \text { for all } \quad f, g \in L^{2}(\mathbb{R})
$$

where $\overline{g(t)}$ denotes the complex conjugate of $g(t)$. The norm, induced by this inner product, is given by

$$
\|f\|^{2}=\langle f, f\rangle .
$$

The Fourier transform of $f \in L^{2}(\mathbb{R})$ is defined as

$$
\mathcal{F} f(\omega)=\int_{-\infty}^{\infty} f(t) e^{-2 \pi i t \omega} d t
$$

For convenience, we will sometimes write $\hat{f}$ for $\mathcal{F} f$.

In order to ensure stable recovery we focus on subspaces of $L^{2}(\mathbb{R})$ which are generated by frames or Riesz bases. A collection of elements $\left\{s_{k}\right\}_{k \in \mathbb{Z}}$ is a frame for its closed linear span if there exist constants $A>0$ and $B<\infty$ such that

$$
A\|f\|^{2} \leq \sum_{k \in \mathbb{Z}}\left|\left\langle f, s_{k}\right\rangle\right| \leq B\|f\|^{2} \quad \text { for all } \quad f \in \overline{\operatorname{span}}\left\{s_{k}\right\}
$$

where $\overline{\text { span }}$ denotes the closed linear span of a set of vectors. The vectors $\left\{s_{k}\right\}_{k \in \mathbb{Z}}$ form a Riesz basis if there exist $A>0$ and $B<\infty$ such that for all sequences $c \in \ell^{2}$

$$
A\|c\|_{\ell^{2}}^{2} \leq\left\|\sum_{k \in \mathbb{Z}} c_{k} s_{k}\right\|^{2} \leq B\|c\|_{\ell^{2}}^{2}
$$

where $\|c\|_{\ell^{2}}^{2}=\sum_{k \in \mathbb{Z}}\left|c_{k}\right|^{2}$ is the squared $\ell^{2}$-norm of $c$. A consequence of the lower inequality is that the basis functions are linearly independent, which means that every function in $\overline{\operatorname{span}}\left\{s_{k}\right\}$ is uniquely specified by its coefficients $c_{k}$.

The fundamental building blocks of the Gabor representation are the so-called Gabor systems. To define a Gabor system, let $a>0$ and $b>0$ be such that $a b=q / p$ with $p$ and $q$ relatively prime, and let $T_{a k}$ and $M_{b l}$, for $k, l \in \mathbb{Z}$, be the translation and modulation operators given by

$$
\begin{aligned}
& T_{a k} f(t)=f(t-a k) ; \\
& M_{b l} f(t)=e^{2 \pi i b l t} f(t) .
\end{aligned}
$$

For $s \in L^{2}(\mathbb{R})$, the Gabor system $\mathcal{G}(s, a, b)$ is a collection $\left\{M_{b l} T_{a k} s(t) ;(k, l) \in \mathbb{Z}^{2}\right\}$. The composition

$$
M_{b l} T_{a k} f(t)=e^{2 \pi i b l t} f(t-a k)
$$

which is a unitary operator, is called a time-frequency shift operator. Many technical details in time-frequency analysis are linked to the commutation law of the translation and modulation operators, namely

$$
M_{b l} T_{a k}=e^{2 \pi i a b k l} T_{a k} M_{b l} .
$$

When $p=1$, the time-frequency shift operators commute, i.e. $M_{b l} T_{a k}=T_{a k} M_{b l}$, because $e^{2 \pi i a b k l}=1$ for all $k, l \in \mathbb{Z}$. One consequence of the commutation rule, which we will use in our exposition, is the relation

$$
\left\langle f, M_{b l-b n} T_{a k-a m} f\right\rangle=e^{2 \pi i a b(l-n) m}\left\langle M_{b n} T_{a m} f, M_{b l} T_{a k} f\right\rangle .
$$

When $p=1$ the exponent is equal to 1 . 


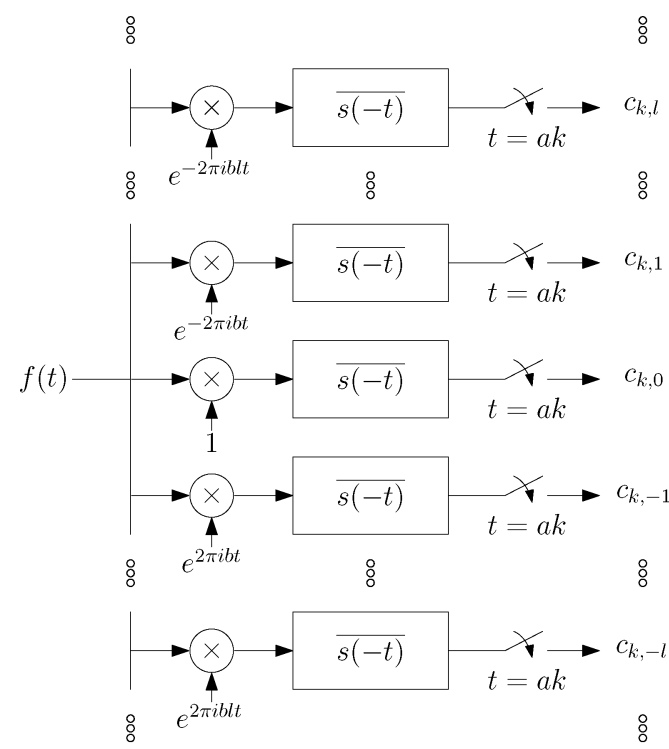

(a) Analysis filter bank

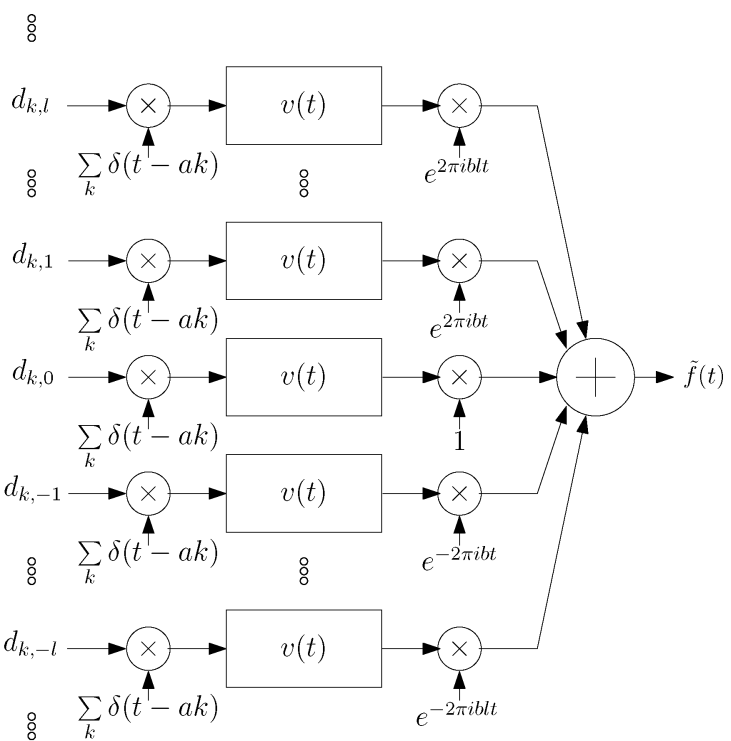

(b) Synthesis filter bank

Fig. 1. Filter-bank representation of the Gabor transform (a) and of the inverse Gabor transform (b). (a) Analysis filter bank. (b) Synthesis filter bank.

For $s \in L^{2}(\mathbb{R})$, the collection $\mathcal{G}(s, a, b)$ is a Riesz basis for its closed linear span if there exist bounds $A>0$ and $B<\infty$ such that

$$
A\|c\|_{\ell^{2}}^{2} \leq\left\|\sum_{k, l \in \mathbb{Z}} c_{k, l} M_{b l} T_{a k} s\right\|^{2} \leq B\|c\|_{\ell^{2}}^{2} \quad c \in \ell^{2}
$$

and is a frame when

$$
A\|f\|^{2} \leq \sum_{k, l \in \mathbb{Z}}\left|\left\langle f, M_{b l} T_{a k} s\right\rangle\right|^{2} \leq B\|f\|^{2}
$$

for all $f \in \overline{\operatorname{span}}\left\{M_{b l} T_{a k} s\right\}$. A necessary condition for $\mathcal{G}(s, a, b)$ to constitute a frame for $L^{2}(\mathbb{R})$ is that $a b \leq 1$. Moreover, if $\mathcal{G}(s, a, b)$ is a frame, then it is a Riesz basis for $L^{2}(\mathbb{R})$ if and only if $a b=1$ [26]. In this paper we focus on the regime $a b>1$, where $\mathcal{G}(s, a, b)$ does not necessarily span $L^{2}(\mathbb{R})$.

We associate a synthesis operator (or reconstruction operator) $S: \ell^{2}\left(\mathbb{Z}^{2}\right) \rightarrow L^{2}(\mathbb{R})$, with $\mathcal{G}(s, a, b)$, defined as

$$
S c=\sum_{k, l \in \mathbb{Z}} c_{k, l} M_{b l} T_{a k} s(t) \quad \text { for every } \quad c \in \ell^{2}\left(\mathbb{Z}^{2}\right) .
$$

The conjugate $S^{*}: L^{2}(\mathbb{R}) \rightarrow \ell^{2}\left(\mathbb{Z}^{2}\right)$ of $S$ is called the analysis operator (or sampling operator), and is given by

$$
S^{*} f=\left\{\left\langle f, M_{b l} T_{a k} s\right\rangle\right\} \quad \text { for every } \quad f \in L^{2}(\mathbb{R}) .
$$

We consider only Gabor systems whose generators come from the so-called Feichtinger algebra $S_{0}$. This set is defined by

$$
S_{0}=\left\{f \in L^{2}(\mathbb{R})\left|\|f\|_{S_{0}}:=\int\right|\left\langle f, M_{\omega} T_{x} \psi\right\rangle \mid d x d \omega<\infty\right\}
$$

where $\psi(t)$ is a Gaussian window. An important property of $S_{0}$ is that if $v(t)$ and $s(t)$ are elements from $S_{0}$ then $\left\{\left\langle v, M_{b l} T_{a k} s\right\rangle\right\}_{k, l \in \mathbb{Z}}$ is an $\ell^{1}\left(\mathbb{Z}^{2}\right)$ sequence. Examples of functions in $S_{0}$ are the Gaussian and B-splines of strictly positive order. The Feichtinger algebra is an extremely useful space of "good" window functions in the sense of time-frequency localization. Rigorous descriptions of $S_{0}$ can be found in [26].

\section{StABLE GABor REPRESENTATIONS}

The Gabor representation of a signal $f(t)$ comprises the set of coefficients $\left\{c_{k, l}\right\}_{k, l \in \mathbb{Z}}$ obtained by inner products with the elements of some Gabor system $\mathcal{G}(s, a, b)$ [26]

$$
c_{k, l}=\left\langle f, M_{b l} T_{a k} s\right\rangle .
$$

This process can be represented as an analysis filter-bank, as shown in Fig. 1(a). Consequently, $s(t)$ is referred to as the analysis window of the transform. If $\mathcal{G}(s, a, b)$ constitutes a frame or Riesz basis for $L^{2}(\mathbb{R})$, then there exists a function $v(t) \in L^{2}(\mathbb{R})$ such that any $f(t) \in L^{2}(\mathbb{R})$ can be reconstructed from the coefficients $\left\{c_{k, l}\right\}_{k, l \in \mathbb{Z}}$ using the formula

$$
f(t)=\sum_{k, l \in \mathbb{Z}} c_{k, l} M_{b l} T_{a k} v(t) .
$$

The Gabor system $\mathcal{G}(v, a, b)$ is the dual frame (Riesz basis) to $\mathcal{G}(s, a, b)$. Consequently, the synthesis window $v(t)$ is referred to as the dual of $s(t)$. The recovery process can be represented as a synthesis filter-bank, as shown in Fig. 1(b).

Generally, there is more than one dual window $v(t)$. It can be shown that any function $v(t)$ satisfying $\left\langle v, M_{l / a} T_{k / b} s\right\rangle=\delta_{k} \delta_{l}$ is a dual window. The canonical dual is given by $v=Q^{-1} s$, where $Q$ is the frame operator associated with $s(t)$, and is defined by $Q f=\sum_{k, l \in \mathbb{Z}}\left\langle f, M_{b l} T_{a k} s\right\rangle M_{b l} T_{a k} s(t)$. There are several ways of finding an inverse of $Q$, including the Janssen representation of $Q$, through the Zak transform method or iteratively using one of several available efficient algorithms [26].

In this paper, we are interested in Gabor systems that do not necessarily span $L^{2}(\mathbb{R})$ but rather only a (shift-and-modulation 
invariant) subspace. A shift-and-modulation (SMI) space is the set $\mathcal{V}$ of all signals that can be expressed in the form (4) with some norm-bounded sequence $c_{k, l}$. In other words, $\mathcal{V}$ is the closed linear span of the Gabor system $\mathcal{G}(v, a, b)$, which in [24] is called the smallest SMI space generated by $\mathcal{G}(v, a, b)$. Our choice of terminology follows from the fact that if $f(t)$ lies in $\mathcal{V}$, then the function $M_{b n} T_{a m} f(t)$ is also an element of $\mathcal{V}$ for every fixed $m, n \in \mathbb{Z}$. Indeed, let $f(t)=\sum_{k, l \in \mathbb{Z}} c_{k, l} M_{b l} T_{a k} v(t)$ for some sequence $c_{k, l}$, then

$$
\begin{aligned}
M_{b n} T_{a m} f(t) & =M_{b m} T_{a n}\left(\sum_{k, l \in \mathbb{Z}} c_{k, l} M_{b l} T_{a k} v(t)\right) \\
& =\sum_{k, l \in \mathbb{Z}} c_{k, l} M_{b n} T_{a m} M_{b l} T_{a k} v(t) \\
& =\sum_{k, l \in \mathbb{Z}} c_{k, l} e^{-2 \pi i a b m l} M_{b(l+n)} T_{a(k+m)} v(t) \\
& =\sum_{k, l \in \mathbb{Z}} c_{k-m, l-n} e^{-2 \pi i a b(l-n) m} M_{b l} T_{a k} v(t) \\
& =\sum_{k, l \in \mathbb{Z}} d_{k, l} M_{b l} T_{a k} v(t) \in \mathcal{V}
\end{aligned}
$$

where $d_{k, l}=c_{k-m, l-n} e^{-2 \pi i a b(l-n) m}$. The same holds for $T_{a m} M_{b n} f(t)$.

Since perfect recovery cannot be guaranteed for every signal in $L^{2}(\mathbb{R})$ in these situations, we have the freedom of choosing the analysis and synthesis windows according to implementation constraints. However, in order for the analysis and synthesis processes to be stable, we would still like to assure that the systems $\mathcal{G}(s, a, b)$ and $\mathcal{G}(v, a, b)$ form frames or Riesz bases for their span. In this section, we give several equivalent characterizations of windows $v(t)$ and sampling intervals $a$ and $b$ such that the Gabor system $\mathcal{G}(v, a, b)$ forms a Riesz basis.

The first characterization of Gabor Riesz bases we consider is stated directly in terms of their generator $v(t)$. It is a simple corollary of a result on Gabor frames for $L^{2}(\mathbb{R})$.

Proposition III.1: Let $v(t) \in S_{0}$ and $a b=q / p$ with $p$ and $q$ relatively prime. The collection $\mathcal{G}(v, a, b)$ is a Riesz basis for its closed linear span if and only if there exist constants $A>0$ and $B<\infty$ such that

$$
A I_{p} \leq V(\omega, x) \leq B I_{p} \quad \text { for almost all }(\omega, x) \in \mathbb{R}^{2}
$$

where $\boldsymbol{I}_{p}$ is the $p \times p$ identity matrix and $\boldsymbol{V}(\omega, x)$ is a $p \times p$ matrix-valued function with entries given by

$\boldsymbol{V}_{r, s}(\omega, x)=$

$$
\frac{1}{b} \sum_{k, l \in \mathbb{Z}} v\left(x-a r-\frac{q k+l}{b}\right) \overline{v\left(x-a s-\frac{l}{b}\right)} e^{-2 \pi i a k \omega}
$$

where $r, s=0, \ldots, p-1 . \mathcal{G}(v, a, b)$ is an orthonormal basis if $A=B=1$.

Proof: By the Ron-Shen duality principle [27], $\mathcal{G}(v, a, b)$ is a Riesz basis (orthonormal basis) for its closed linear span if and only if the system $\mathcal{G}(v, 1 / b, 1 / a)$ is a frame (Parseval frame) for $L^{2}(\mathbb{R})$. The latter holds if and only if there exist constants $A>0$ and $B<\infty$ such that the so-called frame operator $S_{v v}$, defined as $S_{v v} f(t)=\sum_{k, l \in \mathbb{Z}}\left\langle f, M_{l / a} T_{k / b} v\right\rangle M_{l / a} T_{k / b} v(t)$ satisfies

$$
\frac{A}{b} I \leq S_{v v} \leq \frac{B}{b} I
$$

where $I$ is the identity operator on $L^{2}(\mathbb{R})$. It was shown in [26] that, since $1 /(a b)=p / q$, the operator $S_{v v}$ satisfies (7) if and only if (5) is satisfied, which completes the proof.

Note that $\omega$ is a frequency variable associated with the discrete-time variable $k$, and similarly $x$ is a time variable associated with the discrete frequency index $l$. Another valuable observation is that $\boldsymbol{V}(\omega, x)$ is a $(1 / a, 1 / b)$-periodic function. Furthermore, it has been shown in [26] that $V_{r, s}(\omega, x)$ is continuous. Therefore, the lower bound in (5) can be replaced by the requirement that $\operatorname{det}(\boldsymbol{V}(\omega, x))>0$ for all $(\omega, x) \in[0,1 / a) \times[0,1 / b)$.

The next characterization we consider is in terms of the twisted convolution operator. Specifically, the Riesz basis condition implies that $\mathcal{G}(v, a, b)$ is a Riesz basis for its closed linear span if and only if there exist constants $A>0$ and $B<\infty$ such that

$$
A\langle c, c\rangle \leq\left\langle r_{v v} \llbracket c, c\right\rangle \leq B\langle c, c\rangle \text { for all } c \in \ell^{2}\left(\mathbb{Z}^{2}\right)
$$

where the $2 \mathrm{D}$ cross-correlation sequence $r_{v v}[k, l]$ is defined as

$$
r_{v v}[k, l]=\left\langle v, M_{b l} T_{a k} v\right\rangle
$$

The operation $\downarrow$ represents the twisted convolution defined by

$$
(d \measuredangle c)[k, l]=\sum_{m, n \in \mathbb{Z}} d_{m, n} c_{k-m, l-n} e^{-2 \pi i a b(l-n) m} .
$$

Equation (8) follows directly from the definition of Riesz basis (1), by rewriting it in the inner product notation.

When $p=1$, twisted convolution becomes standard convolution, because the exponential term in (10) equals 1 for all $m, n, l \in \mathbb{Z}$. Therefore, $v(t)$ generates a Riesz basis if and only if the twisted convolution (standard convolution when $p=$ 1) operator with kernel $r_{v v}[k, l]$ is bounded and invertible. Invertibility of this operator translates to the invertibility of the sequence $r_{v v}[k, l]$ with respect to $\bigsqcup$ ( $*$, respectively). Proposition III. 1 states then, that this twisted convolution operator is bounded and invertible if and only if the matrix-valued function $V(\omega, x)$ is bounded and invertible for almost all $\omega$ and $x$. Explicitly finding the inverse of a sequence with respect to twisted convolution is not a trivial task. We will address this problem in Appendix A.

Our last representation follows from restating Proposition III.1 using a different, but equivalent, matrix-valued function that involves the cross-correlation sequence $r_{v v}[k, l]$ defined in (9). This new representation was first introduced in [28] to characterize the invertibility of general Gabor frame operators.

Proposition III.2: [28] The matrix-valued function $\boldsymbol{V}(\omega, x)$ of (6) coincides with the matrix-valued function $\boldsymbol{\Phi}(\omega, x)$ whose entries are given by

$$
\boldsymbol{\Phi}_{r, s}(\omega, x)=\sum_{k, l \in \mathbb{Z}} r_{v v}[s-r+p k, l] e^{-2 \pi i a b l r} e^{-2 \pi i(b l x+a k \omega)}
$$


and therefore $\mathcal{G}(v, a, b)$ is a Riesz basis for its closed linear span if and only if there exist constants $A>0$ and $B<\infty$ such that

$$
A \boldsymbol{I}_{p} \leq \boldsymbol{\Phi}(\omega, x) \leq B \boldsymbol{I}_{p}, \quad \text { for almost all }(\omega, x) \in \mathbb{R}^{2} .
$$

In the integer undersampling case $p=1, \Phi(\omega, x)$ of (11) reduces to the scalar function

$$
\Phi(\omega, x)=\sum_{k, l \in \mathbb{Z}} r_{v v}[k, l] e^{-2 \pi i(b l x+a k \omega)}=\left(\mathcal{F} r_{v v}\right)(\omega, x)
$$

where $\mathcal{F} r_{v v}$ is the 2D discrete-time Fourier transform (DTFT) of $r_{v v}[k, l]$. Therefore, in this case condition (12) reduces to

$$
A \leq\left(\mathcal{F} r_{v v}\right)(\omega, x) \leq B \text { for almost all }(\omega, x) \in \mathbb{R}^{2}
$$

for some $A>0$ and $B<\infty$.

The $\boldsymbol{\Phi}$-characterization is of particular interest in our context as it can be used to investigate any twisted convolution operation with a sequence $h \in \ell^{1}\left(\mathbb{Z}^{2}\right)$. Indeed, it was shown in [29] that such an operation is invertible if and only if the matrix-valued function

$$
\boldsymbol{\Phi}_{r, s}^{h}(\omega, x)=\sum_{k, l \in \mathbb{Z}} h_{s-r+p k, l} e^{-2 \pi i a b r l} e^{-2 \pi i(b l x+a k \omega)}
$$

is invertible for almost all $\omega$ and $x$. In fact, in some sense the function $\boldsymbol{\Phi}(\omega, x)$ is to twisted convolution what the DTFT is for convolution. Specifically, we show in Appendix B that for two sequences $c_{k, l}$ and $d_{k, l}$ having $\boldsymbol{\Phi}$-representations $\boldsymbol{\Phi}^{d}(\omega, x)$ and $\boldsymbol{\Phi}^{c}(\omega, x)$, respectively, the matrix-valued function $\boldsymbol{\Phi}^{(c \npreceq d)}(\omega, x)$ associated with the twisted convolution $c \npreceq d$, can be expressed as

$$
\boldsymbol{\Phi}^{(c \natural d)}(\omega, x)=\boldsymbol{\Phi}^{d}(\omega, x) \boldsymbol{\Phi}^{c}(\omega, x) .
$$

We conclude this section with the observation that having a Riesz basis for an SMI space $\mathcal{V}$, it is possible to construct many others using equivalent generating functions.

Proposition III.3: Let $\mathcal{G}(v, a, b)$ be a Riesz basis for its closed linear span $\mathcal{V}$ and $a b=q / p$ with $p$ and $q$ relatively prime. Let

$$
w(t)=\sum_{k, l \in \mathbb{Z}} h_{k, l} M_{b l} T_{a k} v(t)
$$

where $\left\{h_{k, l}\right\}$ is a sequence of weights. Then $\mathcal{G}(w, a, b)$ is an equivalent Riesz basis for $\mathcal{V}$ if and only if there exist constants $A>0$ and $B<\infty$ such that the $(p \times p)$-matrix-valued function $\boldsymbol{\Phi}^{h}(\omega, x)$ of (15) satisfies

$$
A \boldsymbol{I}_{p} \leq \boldsymbol{\Phi}^{h}(\omega, x) \boldsymbol{\Phi}^{h}(\omega, x)^{H} \leq B \boldsymbol{I}_{p}
$$

for almost all $(\omega, x) \in \mathbb{R}^{2}$, where $\boldsymbol{\Phi}^{h}(\omega, x)^{H}$ denotes the conjugate transpose of $\boldsymbol{\Phi}^{h}(\omega, x)$.

Proof: See Appendix C.

In the case of integer undersampling (i.e., when $p=1$ ), $\boldsymbol{\Phi}^{h}(\omega, x)$ becomes a scalar function, which is simply the 2D DTFT of $h_{k, l}$. In this setting, condition (16) becomes

$$
A \leq\left|\Phi^{h}(\omega, x)\right|^{2} \leq B \text { for almost all }(\omega, x) \in \mathbb{R}^{2} .
$$

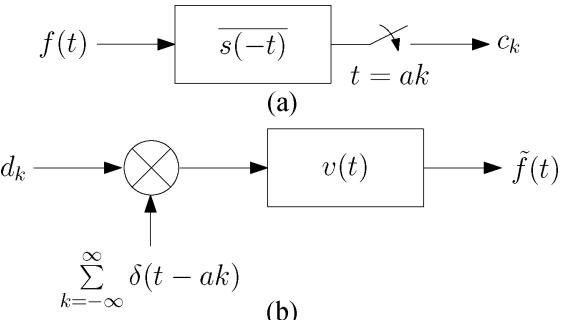

Fig. 2. Sampling (a) and reconstruction (b) with given filters.

\section{SAMPLING AND RECONSTRUCTION IN SHIFT-INVARIANT SPACES}

To address the recovery of a function $f(t)$ from its noninvertible Gabor transform, we will harness several strategies which were initially developed in the context of sampling theory. Specifically, the last two decades have witnessed a substantial amount of research devoted to the problem of recovering a signal $f(t)$ from the equidistant point-wise samples of its filtered version, using a predefined reconstruction filter [10], [11], [30]. As can be seen in Fig. 2, the sampling stage in this setting, corresponds to the central branch in the analysis filter-bank of the Gabor transform shown in Fig. 1(a). Thus, the time-frequency plane is sampled in this scenario only on the lattice $\{(a k, 0)\}_{k \in \mathbb{Z}}$. Similarly, the reconstruction process of Fig. 2 can be identified with the central branch of the synthesis filter-bank of Fig. 1(b).

The main goal in this setting is to produce a set of expansion coefficients $\left\{d_{k}\right\}$ by processing the samples $\left\{c_{k}\right\}$, such that the recovered signal $\tilde{f}(t)$ possesses certain desired properties. In this section, we briefly review three methods for treating this problem, each based on a different design criterion. For more detailed explanations and a review of other methods, we refer the reader to [11]-[13], [30], [31]. In the following sections, we will extend these results to the Gabor scenario.

For simplicity, we assume here that $a=1$. The reconstruction process of Fig. 2 can be written in operator notation as $\tilde{f}=V d$, where $V: \ell^{2} \rightarrow L^{2}(\mathbb{R})$ is the synthesis operator associated with the functions $\{v(t-k)\}_{k \in \mathbb{Z}}$, defined as

$$
V d=\sum_{k \in \mathbb{Z}} d_{k} v(t-k)=\sum_{k \in \mathbb{Z}} d_{k} T_{k} v(t) \text { for every } d \in \ell^{2}(\mathbb{Z})
$$

Similarly, since $c_{k}=\langle f(t), s(t-k)\rangle$, the sequence of samples $\left\{c_{k}\right\}$ are obtained by applying the synthesis operator $S^{*}$, which is the conjugate of the analysis operator $S$ associated with the functions $\{s(t-k)\}_{k \in \mathbb{Z}}$

$S^{*} f=\{\langle f(t), s(t-k)\rangle\}=\left\{\left\langle f, T_{k} s\right\rangle\right\}$ for every $f \in L^{2}(\mathbb{R})$.

We will refer to $\mathcal{S}=\overline{\operatorname{span}}\{s(t-k)\}$ and $\mathcal{V}=\overline{\operatorname{span}}\{v(t-k)\}$ as the sampling and reconstruction spaces, respectively. Spaces of this type are called shift-invariant (SI).

As in the Gabor transform, we focus on cases where the sets of functions $\{s(t-n)\}$ and $\{v(t-n)\}$ constitute Riesz bases for 
their span. It is well known [32] that this holds if, for example, $v(t)$ satisfies

$$
A \leq \phi_{V V}(\omega) \leq B \text { for almost all } \omega \in \mathbb{R}
$$

for some $A>0$ and $B<\infty$, where

$$
\phi_{V V}(\omega)=\frac{1}{2 \pi} \sum_{k \in \mathbb{Z}}|\hat{v}(\omega-k)|^{2} .
$$

The function $\phi_{V V}(\omega)$ is the DTFT of the auto-correlation sequence

$$
r_{v v}[n]=\langle v(t), v(t-n)\rangle=(v(t) * \overline{v(-t)})(n)
$$

where $\hat{v}(\omega)$ is the Fourier transform of $v(t)$. In other words, $\{v(t-n)\}$ is a Riesz basis if and only if the sequence $r_{v v}[n]$ is bounded and invertible in the convolution algebra $\ell^{1}(\mathbb{Z}, *)$. In particular, the functions $\{v(t-n)\}$ form an orthonormal basis if and only if $A=B=1$. Notice the analogy with condition (12) [and (14) in the case $p=1$ ], which was developed for Gabor systems.

\section{A. Consistent Reconstruction}

Perhaps the most intuitive demand on the recovered signal $\tilde{f}(t)$ is that it produces the same sequence of samples $\left\{c_{k}\right\}$ when it is reinjected into the sampling device of Fig. 2(a), namely

$$
\langle\tilde{f}(t), s(t-k)\rangle=c_{k}=\langle f(t), s(t-k)\rangle
$$

for all $k \in \mathbb{Z}$. This consistency requirement was first introduced in [12] in the context of sampling in SI spaces and then generalized to arbitrary spaces in [31] and [33]. There, it was shown that consistent reconstruction is possible under the direct-sum condition $\mathcal{S}^{\perp} \oplus \mathcal{V}=L^{2}(\mathbb{R})$, where $\oplus$ denotes a sum of two subspaces that intersect only at the zero vector. This means that $\mathcal{S}^{\perp}$ and $\mathcal{V}$ are disjoint and together span the space $L^{2}(\mathbb{R})$.

In the SI setting, the direct-sum condition translates into the simple requirement that [17]

$$
\left|\phi_{S V}(\omega)\right|>A, \quad \text { for almost all } \omega \in \mathbb{R}
$$

for some positive constant $A$, where

$$
\phi_{S V}(\omega)=\frac{1}{2 \pi} \sum_{k \in \mathbb{Z}} \overline{\hat{s}(\omega-k)} \hat{v}(\omega-k)
$$

is the DTFT of the cross-correlation sequence $r_{s v}[n]=$ $\langle s(t), v(t-n)\rangle$. Under this condition, reconstruction can be obtained by convolving the sample sequence $\left\{c_{k}\right\}$ with the filter $h_{\text {con }}$, whose DTFT is given by [12], [33], [34]

$$
H_{\mathrm{con}}(\omega)=\frac{1}{\phi_{S V}(\omega)}
$$

to obtain the sequence of expansion coefficients ${ }^{1}\left\{d_{k}\right\}$.

\footnotetext{
${ }^{1}$ When assigning $H_{\text {con }}(\omega)$ in (21) we set it to zero whenever $\phi_{S V}(\omega)$ is zero. It can happen that $\phi_{S V}(\omega)$ is zero for some $\omega$, since the condition (19) holds for almost all $\omega$, and not all. Such an assignment rule will also hold for other $H$ throughout the presentation, whenever it is defined by $\phi$ that is almost everywhere positive.
}

If $\mathcal{S}$ and $\mathcal{V}$ are two arbitrary subspaces of $L^{2}(\mathbb{R})$ satisfying $\mathcal{S}^{\perp} \oplus \mathcal{V}=L^{2}(\mathbb{R})$ (namely not necessarily SI spaces), spanned by the functions $\left\{s_{n}(t)\right\}$ and $\left\{v_{n}(t)\right\}$, respectively, then the sequence of expansion coefficients $d$ can be obtained by applying the operator

$$
\mathcal{H}_{\text {con }}=\left(S^{*} V\right)^{-1}
$$

on the sequence of samples $c$, where $S$ and $V$ are the synthesis operators associated with $\left\{s_{n}(t)\right\}$ and $\left\{v_{n}(t)\right\}$, respectively. The direct-sum requirement guarantees that $S^{*} V: \ell^{2} \rightarrow$ $\ell^{2}$ is continuously invertible. In the next sections, we will use this latter characterization to develop a consistent reconstruction procedure for noninvertible Gabor transforms.

\section{B. Minimax Regret Reconstruction}

A drawback of the consistency approach is that the fact that $f(t)$ and $\tilde{f}(t)$ yield the same samples does not necessarily imply that $\tilde{f}(t)$ is close to $f(t)$. Indeed, for a signal $f(t)$ not in $\mathcal{V}$, the norm of the resulting reconstruction error $\tilde{f}(t)-f(t)$ can be arbitrarily large, if $\mathcal{S}$ is nearly orthogonal to $\mathcal{V}$.

To directly control the reconstruction error, it is important to notice that $\tilde{f}(t)$ is restricted to lie in $\mathcal{V}$ by construction. Therefore, the best possible recovery is the orthogonal projection of $f(t)$ onto $\mathcal{V}$, namely $\tilde{f}=P_{\mathcal{V}} f$, a fact that follows from the projection theorem. This solution cannot be generated in general, because we do not know $f(t)$ but rather only the sequence of samples $\left\{c_{k}\right\}$ it produced. The difference between the squarednorm error of any recovery $\tilde{f}(t)$ and the smallest possible error, which is $\left\|f-P_{\mathcal{V}} f\right\|^{2}=\left\|P_{\mathcal{V}} f \mid\right\|^{2}$, is called the regret [35], [36]. The regret depends in general on $f(t)$ and therefore cannot be minimized uniformly for all $f(t)$. Instead, the authors in [13] proposed minimizing the worst-case regret over all boundednorm signals $f(t)$ that are consistent with the given samples. This results in the problem

$$
\min _{\tilde{f} \in \mathcal{V}} \max _{f \in \mathcal{B}}\|\tilde{f}-f\|^{2}-\left\|P_{\mathcal{V}} f\right\|^{2}
$$

where $\mathcal{B}=\left\{f: S^{*} f=c,\|f\| \leq L\right\}$ is the set of feasible signals.

It was shown in [13] that the minimax-regret reconstruction can be obtained by filtering the samples $c_{k}$ with the filter $h_{\mathrm{mx}}$ whose DTFT is given by

$$
H_{\mathrm{mx}}(\omega)=\frac{\phi_{V S}(\omega)}{\phi_{S S}(\omega) \phi_{V V}(\omega)}
$$

where $\phi_{V S}(\omega), \phi_{S S}(\omega)$ and $\phi_{V V}(\omega)$ are as in (20) with the corresponding substitution of the generators $v(t)$ and $s(t)$. Note that the solution is independent of the bound $L$ appearing in the definition of $\mathcal{B}$.

If the sampling and reconstruction functions form Riesz bases for arbitrary spaces $\mathcal{S}$ and $\mathcal{V}$ (not necessarily SI), then the sequence of expansion coefficients $d$ can be obtained by applying the operator

$$
\mathcal{H}_{\mathrm{mx}}=\left(V^{*} V\right)^{-1} S^{*} V\left(S^{*} S\right)^{-1}
$$

on the sequence of samples $c$. The operators $V^{*} V$ and $S^{*} S$ are guaranteed to be continuously invertible due to the Riesz basis 
assumption. This more general characterization will be used later to obtain a minimax-regret recovery method in the setting of SMI spaces.

\section{Subspace-Prior Reconstruction}

The consistent reconstruction approach leads to perfect recovery for input signals that lie in the reconstruction space $\mathcal{V}$ [12]. The minimax-regret method, on the other hand, leads to the best possible approximation $\tilde{f}=P \mathcal{V} f$ for signals $f(t)$ lying in the sampling space $\mathcal{S}$ [13]. Therefore, the two methods can be thought of as emerging from the prior that $f(t)$ lies in a certain subspace $\mathcal{W}$ of $L^{2}(\mathbb{R})$, where $\mathcal{W}=\mathcal{V}$ in the consistent strategy and $\mathcal{W}=\mathcal{S}$ in the minimax-regret approach. In practice, though, it is often desirable to choose the sampling and reconstruction spaces according to implementation constraints and not to reflect our prior knowledge on the typical signals entering our sampling device. Thus, commonly neither constitutes a subspace prior $\mathcal{W}$, which is good in the sense that $\left\|f-P_{\mathcal{W}} f\right\|$ is small for most signals in our application.

A generalization of these two methods results by assuming that $f \in \mathcal{W}$ where $\mathcal{W}=\overline{\operatorname{span}}\{w(t-k)\}$ for a generator $w(t)$, which may be different than $s(t)$ and $v(t)$. If the subspace $\mathcal{W}$ satisfies the direct-sum condition $\mathcal{S}^{\perp} \oplus \mathcal{W}=L^{2}(\mathbb{R})$, then the solution $\tilde{f}=P_{\mathcal{V}} f$ can be generated by filtering the sample sequence $c_{k}$ with [13]

$$
H_{\mathrm{sub}}(\omega)=\frac{\phi_{V W}(\omega)}{\phi_{S W}(\omega) \phi_{V V}(\omega)}
$$

where $\phi_{V W}(\omega), \phi_{S W}(\omega)$ and $\phi_{V V}(\omega)$ are as in (20) with the appropriate substitution of $v(t), s(t)$, and $w(t)$.

For general sampling, reconstruction and prior subspaces $\mathcal{S}$, $\mathcal{V}$ and $\mathcal{W}$, the coefficient sequence $d$ can be obtained by applying the transformation

$$
\mathcal{H}_{\text {sub }}=\left(V^{*} V\right)^{-1} V^{*} W\left(S^{*} W\right)^{-1}
$$

on the sample sequence $c$, where $W$ is the synthesis operator associated with the prior functions $\left\{w_{n}(t)\right\}$. Again, this general formulation will be used in the context of SMI spaces to derive a subspace-prior recovery technique.

\section{INTEGER UNDERSAMPLING}

In this section, we address the problem of recovering a signal $f(t)$ from its noninvertible Gabor transform coefficients $\left\{c_{k, l}\right\}$, given by (3), using a pre-specified synthesis window $v(t)$. We focus on prior-free approaches that do not take into account any knowledge on the signal $f(t)$. Specifically, here we employ the consistency and minimax-regret methods discussed in the previous section to the Gabor scenario. To emphasize the commonalities with respect to the SI sampling case, and to retain simplicity, we begin the discussion with the case of integer undersampling $(p=1)$. In the next section we generalize the results to arbitrary $p$.

\section{A. Consistent Synthesis}

In the Gabor transform, the sampling (analysis) space $\mathcal{S}$ is spanned by the Gabor system $\mathcal{G}(s, a, b)$ and the reconstruction (synthesis) space $\mathcal{V}$ is the span of $\mathcal{G}(v, a, b)$. As discussed in Section IV-A, consistent reconstruction is possible if $\mathcal{S}^{\perp} \oplus \mathcal{A}=$ $L^{2}(\mathbb{R})$. For SI spaces, this direct-sum condition translates to the requirement that the cross-correlation sequence $\{\langle s(t), v(t-$ $n)\rangle\}_{n \in \mathbb{Z}}$ has an inverse in the convolution algebra $\ell^{1}\left(\mathbb{Z}^{2}, *\right)$. A similar condition is true in the setting of Gabor spaces.

The next proposition characterizes the class of pairs of analysis and synthesis windows satisfying the direct-sum requirement in the integer undersampling regime.

Proposition V.1: Assume that $\mathcal{G}(s, a, b)$ and $\mathcal{G}(v, a, b)$ are Riesz sequences that span the spaces $\mathcal{S}$ and $\mathcal{V}$, respectively, and $a b=q \in \mathbb{N}$. Then $\mathcal{S}^{\perp} \oplus \mathcal{V}=L^{2}(\mathbb{R})$ if and only if the function $\Phi^{s v}(\omega, x)$, defined as

$$
\Phi^{s v}(\omega, x)=\sum_{k, l \in \mathbb{Z}} r_{s v}[k, l] e^{-2 \pi i(b l x+a k \omega)}=\left(\mathcal{F} r_{s v}\right)(\omega, x)
$$

is nonzero for almost all $(\omega, x) \in[0,1 / a) \times[0,1 / b)$. Here

$$
r_{s v}[k, l]=\left\langle v, M_{b n} T_{a m} s\right\rangle
$$

is the Gabor transform of the synthesis window $v(t)$.

Proof: It was shown in [31], for general Hilbert spaces, that if $\mathcal{S}$ and $\mathcal{V}$ are spanned by Riesz bases $\mathcal{G}(s, a, b)$ and $\mathcal{G}(v, a, b)$, respectively, then $\mathcal{S}^{\perp} \oplus \mathcal{V}=L^{2}(\mathbb{R})$ if and only if the operator $S^{*} V$ is continuously invertible on $\ell^{2}$. Here, $S^{*}$ and $V$ are the analysis and synthesis operators associated with $\mathcal{G}(s, a, b)$ and $\mathcal{G}(v, a, b)$, respectively. By definition, for any sequence $c \in$ $\ell^{2}\left(\mathbb{Z}^{2}\right)$

$$
\begin{aligned}
\left(S^{*} V c\right)[k, l]= & \left\langle\sum_{m, n \in \mathbb{Z}} c_{m, n} M_{b n} T_{a m} v, M_{b l} T_{a k} s\right\rangle \\
= & \sum_{m, n \in \mathbb{Z}} c_{m, n}\left\langle v, M_{b l-b n} T_{a k-a m} s\right\rangle \\
& \cdot e^{-2 \pi i a b(l-n) m} \\
= & \sum_{m, n \in \mathbb{Z}} c_{k-m, l-n}\left\langle v, M_{b n} T_{a m} s\right\rangle \\
= & \left(r_{s v} * c\right)[k, l]
\end{aligned}
$$

where the third equality follows from the fact $a b \in \mathbb{N}$, and hence $e^{-2 \pi i a b(l-n) m}=1$. Therefore, the operator $S^{*} V$ is simply a 2D convolution operator with kernel $r_{s v}[k, l]=\left\langle v, M_{b n} T_{a m} s\right\rangle$ and $S^{*} V$ is invertible if and only if $r_{s v}[k, l]$ is invertible in the convolution algebra $\ell^{1}\left(\mathbb{Z}^{2}, *\right)$. As shown in Section III, this sequence has a representation $\Phi^{s v}(\omega, x)$, defined by (15), which is its $2 \mathrm{D}$ DTFT in the case $p=1$. A sequence is invertible with respect to convolution if and only if its DTFT has no zeros. Therefore, $r_{s v}[k, l]$ is invertible if and only if $\Phi^{s v}(\omega, x) \neq 0$ implying that $\mathcal{S}^{\perp} \oplus \mathcal{V}=L^{2}(\mathbb{R})$ if and only if $\Phi^{s v}(\omega, x) \neq 0$.

Assuming that indeed $\mathcal{S}^{\perp} \oplus \mathcal{A}=L^{2}(\mathbb{R})$, we know from Section IV-A that to obtain a consistent recovery, we must apply the operator $\mathcal{H}_{\text {con }}=\left(S^{*} V\right)^{-1}$ on the coefficients $\left\{c_{k, l}\right\}$ prior to synthesis. In the proof of Proposition V.1, we showed that $S^{*} V$ is a $2 \mathrm{D}$ convolution operator with the kernel $r_{s v}[k, l]$ of (27). Therefore, $\left(S^{*} V\right)^{-1}$ corresponds to filtering the Gabor coefficients with the filter $h_{\text {con }}$ whose 2D DTFT is given by

$$
H_{\text {con }}(\omega, x)=\frac{1}{\Phi^{s v}(\omega, x)} \text {. }
$$


This filter is well defined by Proposition V.1 since we assumed that the spaces generated by $s(t)$ and $v(t)$ satisfy the direct sum condition.

During the operations of analysis and pre-processing of the Gabor coefficients $c_{k, l}$, we in fact compute a dual Riesz basis for the reconstruction space $\mathcal{V}$. In case the synthesis and analysis spaces are the same, namely $\mathcal{S}=\mathcal{V}$, we compute the orthogonal dual basis. However, when the spaces are different we compute a general (oblique) dual Riesz basis for $\mathcal{V}$.

Proposition V.2: Let $\mathcal{G}(s, a, b)$ and $\mathcal{G}(v, a, b)$ be Riesz sequences that span the spaces $\mathcal{S}$ and $\mathcal{V}$, respectively, where $a b$ is an integer, and assume that $\mathcal{S}^{\perp} \oplus \mathcal{V}=L^{2}(\mathbb{R})$. Then a dual Riesz basis for the space $\mathcal{V}$ is $\mathcal{G}(g, a, b)$ with

$$
g(t)=\sum_{m, n \in \mathbb{Z}} \overline{h_{\mathrm{con}}[m, n]} T_{-a m} M_{-b n} s(t) \in \mathcal{S}
$$

where $h_{\text {con }}[m, n]$ is the inverse of $r_{s v}[k, l]$ with respect to $*$.

Proof: Any signal in $\mathcal{V}$ can be recovered from the corrected coefficients $d_{k, l}=\left(h_{\text {con }} * c\right)[k, l]$ via $f(t)=\sum_{k, l \in \mathbb{Z}} d_{k, l} M_{b l} T_{a k} v(t)$, where $c_{k, l}$ is as in (3). Therefore, we may view this sequence as the coefficients in a basis expansion. To obtain the corresponding basis we note that by combining the effects of the analysis window $s(t)$ and the correction filter $H_{\text {con }}$ of (32), the expansion coefficients can be equivalently expressed as $d_{k, l}=\left\langle f, M_{b l} T_{a k} g\right\rangle$ where

$$
g(t)=\sum_{m, n \in \mathbb{Z}} \overline{h_{\mathrm{con}}[m, n]} T_{-a m} M_{-b n} s(t) \in \mathcal{S} .
$$

Indeed

$$
\begin{aligned}
\langle f, & \left.M_{b l} T_{a k} g\right\rangle \\
& =\left\langle f, \sum_{m, n \in \mathbb{Z}} \overline{h_{\mathrm{con}}[m, n]} M_{b l} T_{a k} T_{-a m} M_{-b n} s\right\rangle \\
& =\left\langle f, \sum_{m, n \in \mathbb{Z}} \overline{h_{\mathrm{con}}[m, n]} e^{2 \pi i a b(k-m) n} M_{b l-b n} T_{a k-a m} s\right\rangle \\
& =\sum_{m, n \in \mathbb{Z}} h_{\mathrm{con}}[m, n]\left\langle f, M_{b l-b n} T_{a k-a m} s\right\rangle
\end{aligned}
$$

and the last equality is due to the fact that $a b \in \mathbb{N}$, and therefore the exponent equals 1 . Noting that $\left\langle f, M_{b l-b n} T_{a k-a m} s\right\rangle=$ $c_{k-m, l-n},(34)$ becomes

$$
\begin{aligned}
\left\langle f, M_{b l} T_{a k} g\right\rangle & =\sum_{m, n \in \mathbb{Z}} h_{\mathrm{con}}[m, n] c_{k-m, l-n} \\
& =\left(h_{\mathrm{con}} * c\right)[k, l]=d_{k, l} .
\end{aligned}
$$

Therefore, any $f \in \mathcal{V}$ can be written as

$$
f(t)=\sum_{k, l \in \mathbb{Z}}\left\langle f, M_{b l} T_{a k} g\right\rangle M_{b l} T_{a k} v(t) .
$$

It can be easily verified, by Proposition III.3, that $\mathcal{G}(g, a, b)$ is an equivalent Riesz basis for $\mathcal{S}$. Furthermore

$$
\left\langle M_{b l} T_{a k} v, M_{b n} T_{a m} g\right\rangle=\delta_{m-k} \delta_{n-l}
$$

implying that $\mathcal{G}(g, a, b)$ is a dual Riesz basis to $\mathcal{G}(v, a, b)$.

\section{B. Minimax Regret Synthesis}

We now develop a minimax-regret recovery method, similar to the SI case of Section IV-B. Specifically, we seek a recovery $\tilde{f}(t)$ for which the worst-case regret over all bounded-norm signals $f(t)$ consistent with the given Gabor coefficients $\left\{c_{k, l}\right\}$, is minimal. The minimax-regret reconstruction can be obtained by applying the operator $\mathcal{H}_{\mathrm{mx}}$ of (24) on the Gabor coefficients $c_{k, l}$ prior to synthesis.

When $p=1$ we have shown in Section V-A that the operators $V^{*} V, S^{*} V$ and $S^{*} S$ correspond to 2D convolutions with the kernels $r_{v v}[k, l], r_{s v}[k, l]$ and $r_{s s}[k, l]$, respectively, which are given by (27) with the appropriate substitution of $s(t)$ and $v(t)$. Therefore, the minimax-regret recovery is obtained by filtering the Gabor coefficients $c_{k, l}$ with the 2D filter $h_{\mathrm{mx}}$, whose DTFT is given by

$$
H_{\mathrm{mx}}(\omega, x)=\frac{\Phi^{s v}(\omega, x)}{\Phi^{s s}(\omega, x) \Phi^{v v}(\omega, x)} .
$$

Here, $\Phi^{s v}(\omega, x), \Phi^{s s}(\omega, x)$, and $\Phi^{v v}(\omega, x)$ are the 2D DTFTs of $r_{s v}[k, l], r_{s s}[k, l]$ and $r_{v v}[k, l]$, respectively. This filter is well defined by Proposition III.2 since we assumed that $s(t)$ and $v(t)$ generate Riesz bases for their span.

\section{Efficient Implementation}

The two reconstruction approaches discussed above are based on 2D filtering of the Gabor transform $c_{k, l}$ prior to synthesis. A significant reduction in computation can be achieved when the 2D correction filter is a separable function of $k$ and $l$, namely when $h_{k, l}=u_{k} v_{l}$ for two sequences $u_{k}$ and $v_{k}$. In these situations, one can first apply the 1D filter $u_{k}$ on each of the rows of $c_{k, l}$ (i.e., along the time direction), and then apply the 1D filter $v_{l}$ on each of the columns (along the frequency direction). If, for example, $h_{k, l}$ is a separable finite-impulse-response (FIR) filter with $N \times N$ nonzero coefficients, then direct application of it requires $N^{2}$ multiplications per output coefficient, whereas only $2 N$ multiplications suffice when implementing it using two 1D filtering operations.

Separable correction filters emerge when the cross-correlation sequences involved are separable functions of $k$ and $l$. One such example is the setting in which $s(t)$ and $v(t)$ are Gaussian windows with variances $\sigma_{s}^{2}$ and $\sigma_{v}^{2}$, respectively, and $a b \sigma_{s}^{2} /\left(\sigma_{s}^{2}+\sigma_{v}^{2}\right)$ is an integer (recall that we also require that $a b$ be an integer). Then $r_{s v}[k, l], r_{s s}[k, l]$, and $r_{v v}[k, l]$ are all separable functions of $k$ and $l$, so that both the consistent and the minimax-regret filters are separable. More details on noninvertible Gaussian-window Gabor transforms are given in Section VIII.

\section{Rational Undersampling}

We now generalize the results of the previous section to the case where the product $a b$ is not an integer, but rather some rational number $q / p$ with $p$ and $q$ relatively prime. The main difficulty here is the fact that the time-frequency shift operators do not commute when $p \neq 1$. Therefore, instead of standard convolution we will be faced with a twisted convolution, which is a noncommutative operation. This makes the techniques from Fourier theory inapplicable in a straightforward manner. 


\section{E. Consistent Synthesis}

Obtaining a reconstruction $\tilde{f}(t)$, which is consistent with the Gabor representation $c_{k, l}$ of $f(t)$, is possible if $\mathcal{S}^{\perp} \oplus \mathcal{V}=$ $L^{2}(\mathbb{R})$. As we have seen in Proposition V.1, in the integer undersampling case $p=1$ the direct sum condition translates to the requirement that the cross-correlation sequence $r_{s v}[k, l]$ be invertible in the convolution algebra $\ell^{1}\left(\mathbb{Z}^{2}, *\right)$. In the setting of rational undersampling, we have the following.

Proposition VI.1: Assume that $\mathcal{G}(s, a, b)$ and $\mathcal{G}(v, a, b)$ are Riesz sequences that span the spaces $\mathcal{S}$ and $\mathcal{V}$, respectively, and $a b=q / p$ with $p$ and $q$ relatively prime. Then $\mathcal{S}^{\perp} \oplus \mathcal{V}=L^{2}(\mathbb{R})$ if and only if the $(p \times p)$-matrix-valued function $\boldsymbol{\Phi}^{s v}(\omega, x)$ with entries $m, n=0, \ldots, p-1$ defined as

$\boldsymbol{\Phi}_{m, n}^{s v}(\omega, x)=\sum_{k, l \in \mathbb{Z}} r_{s v}[n-m+p k, l] e^{-2 \pi i a b l m} e^{-2 \pi i(b l x+a k \omega)}$

is invertible for almost all $(\omega, x) \in[0,1 / a) \times[0,1 / b)$. This is equivalent to $\operatorname{det}(\boldsymbol{\Phi}(\omega, x)) \neq 0$ for all $(\omega, x)$.

Proof: The proof is similar to that of Proposition V.1, with the difference that the standard convolution becomes twisted convolution. This is due to the fact that the exponent in (28) no longer equals 1 , since the product $a b$ is rational. Therefore

$$
\begin{aligned}
\left(S^{*} V c\right)[k, l] & =\sum_{m, n \in \mathbb{Z}} c_{k-m, l-n}\left\langle v, M_{b n} T_{a m} s\right\rangle e^{-2 \pi i a b(k-m) n} \\
& =\left(r_{s v}\lfloor c)[k, l],\right.
\end{aligned}
$$

where the operation $\downarrow$ is defined in (10). Consequently, $S^{*} V$ is a twisted convolution operator with kernel

$$
r_{s v}[k, l]=\left\langle v, M_{b l} T_{a k} s\right\rangle
$$

and $S^{*} V$ is invertible if and only if $r_{s v}[k, l]$ is invertible in the twisted convolution algebra $\ell^{1}\left(\mathbb{Z}^{2}, \emptyset\right)$. As shown in Section III, this sequence has a representation $\Phi^{s v}(\omega, x)$ defined by (15) and so is invertible if and only if this matrix is invertible. Therefore, $\mathcal{S}^{\perp} \oplus \mathcal{V}=L^{2}(\mathbb{R})$ if and only if $\boldsymbol{\Phi}^{s v}(\omega, x)$ is invertible almost everywhere.

Note that for $p=1$, the above proposition reduces to Proposition V.1. When $p \neq 1$, we conclude from Proposition VI.1 that the direct sum condition translates to the requirement that $r_{s v}[k, l]$ be invertible in the twisted convolution algebra, which can be checked by analyzing its $\boldsymbol{\Phi}$-representation. An alternative method for checking whether $r_{s v}[k, l]$ is invertible with respect to $\downarrow$, is presented in Appendix A. It involves only the sequence $r_{s v}[k, l]$ without introducing the continuous variables $\omega$ and $x$, making it more attractive in some cases.

As in Section V-A, to obtain a consistent recovery $\tilde{f}(t)$, we have to apply the operator $\mathcal{H}_{\text {con }}=\left(S^{*} V\right)^{-1}$ to the Gabor coefficients $c_{k, l}$. However, in contrast to the case $p=1$, where $\mathcal{H}_{\text {con }}$ was a standard convolution operator, here it corresponds to a twisted convolution operation. This is due to the fact that time-frequency shift operators do not commute for $p \neq 1$. Specifically, in the proof of Proposition VI.1, it was shown that $S^{*} V$ corresponds to twisted convolution with $r_{s v}[k, l]$. Therefore, $\left(S^{*} V\right)^{-1}$ corresponds to twisted convolution with the sequence $r_{s v}^{-1}[k, l]$, which is the inverse of $r_{s v}[k, l]$ in the twisted convolution algebra $\ell^{1}\left(\mathbb{Z}^{2}, \downarrow\right)$. This inverse exists, since we assumed that the spaces generated by $s(t)$ and $v(t)$ satisfy the direct-sum condition. In Appendix A we will show how to construct it.

One can write the twisted convolution relation between the Gabor transform $c_{k, l}$ and the expansion coefficients $d_{k, l}$ in terms of their $\boldsymbol{\Phi}$-representations. Specifically, since $d=\left(S^{*} V\right)^{-1} c$, we have $c_{k, l}=\left(r_{s v} \downarrow d\right)[k, l]$ and, therefore

$$
\boldsymbol{\Phi}^{c}(\omega, x)=\boldsymbol{\Phi}^{d}(\omega, x) \boldsymbol{\Phi}^{s v}(\omega, x)
$$

where $\boldsymbol{\Phi}^{c}(\omega, x), \boldsymbol{\Phi}^{d}(\omega, x)$ and $\boldsymbol{\Phi}^{s v}(\omega, x)$ are the $p \times p$-matrix-valued $\boldsymbol{\Phi}$-representations of the sequences $c_{k, l}, d_{k, l}$ and $r_{s v}[k, l]$, respectively, defined in (11). Therefore, to obtain the sequence $d_{k, l}$ from the Gabor coefficients $c_{k, l}$, we apply a twisted convolution filter, whose $\boldsymbol{\Phi}$ function is

$$
\boldsymbol{H}_{\mathrm{con}}(\omega, x)=\boldsymbol{\Phi}^{s v}(\omega, x)^{-1} \text {. }
$$

The twisted convolution operation can be modeled as a filter bank which is specified by the convolutional inverse of $r_{s v}[k, l]$, as we show in Appendix A.

During the operations of sampling and pre-processing of the samples $c_{k, l}$ we in fact compute a dual Riesz basis for the synthesis space $\mathcal{V}$. If the synthesis and analysis spaces are the same, namely $\mathcal{S}=\mathcal{V}$, we compute the orthogonal dual basis. However, when the spaces are different we compute a general (oblique) dual Riesz basis for $\mathcal{V}$.

Proposition VI.2: Let $\mathcal{G}(s, a, b)$ and $\mathcal{G}(v, a, b)$ be Riesz sequences that span the spaces $\mathcal{S}$ and $\mathcal{V}$, respectively, and $a b=$ $q / p$ with $p$ and $q$ relatively prime. Assume that $\mathcal{S}^{\perp} \oplus \mathcal{V}=$ $L^{2}(\mathbb{R})$. Then a dual Riesz basis for the space $\mathcal{V}$ is $\mathcal{G}(g, a, b)$ with

$$
g(t)=\sum_{m, n \in \mathbb{Z}} \overline{h_{\mathrm{con}}[m, n]} T_{-a m} M_{-b n} s(t) \in \mathcal{S}
$$

where $h_{\text {con }}[m, n]$ is the inverse of $r_{s v}[k, l]$ with respect to $t$.

Proof: The proof is analogous to the proof of Proposition V.2. The only change is that the standard convolution becomes twisted convolution. This is because the time-frequency shifts do not commute when $a b$ is not an integer. Therefore

$$
\begin{aligned}
\left\langle f, M_{b l} T_{a k} g\right\rangle= & \sum_{m, n \in \mathbb{Z}} h_{\operatorname{con}}[m, n] e^{-2 \pi i a b(k-m) n} \\
& \cdot\left\langle f, M_{b l-b n} T_{a k-a m} s\right\rangle \\
= & \sum_{m, n \in \mathbb{Z}} h_{\operatorname{con}}[m, n] e^{-2 \pi i a b(k-m) n} c_{k-m, l-n} \\
= & \left(h_{\operatorname{con}}\lfloor c)[k, l]=d_{k, l}\right.
\end{aligned}
$$

and any $f \in \mathcal{V}$ can be written as

$$
f(t)=\sum_{k, l \in \mathbb{Z}}\left\langle f, M_{b l} T_{a k} g\right\rangle M_{b l} T_{a k} v(t) .
$$

It can be easily verified, by Proposition III.3, that $\mathcal{G}(g, a, b)$ is an equivalent Riesz basis for $\mathcal{S}$. Now, for it to be a dual Riesz basis to $\mathcal{G}(v, a, b)$ we need to check that

$$
\left\langle M_{b l} T_{a k} v, M_{b n} T_{a m} g\right\rangle=\delta_{m-k} \delta_{n-l} .
$$


Let $\omega=e^{2 \pi i a b}$ and $h_{m, n}=h_{\mathrm{con}}[m, n]$. Then,

$$
\left\langle M_{b l} T_{a k} v, M_{b n} T_{a m} g\right\rangle=\omega^{(l-n) k}\left\langle v, M_{b(n-l)} T_{a(m-k)} g\right\rangle .
$$

Using the fact that

$$
M_{b n} T_{a m} g(t)=\sum_{x, y \in \mathbb{Z}} \overline{h_{x, y}} \omega^{(m-x) y} M_{b(n-y)} T_{a(m-x)} s(t)
$$

and that $h_{\text {con }}$ is the inverse of $r_{s v}$ with respect to $t$, we have

$$
\begin{aligned}
\langle v & \left.M_{b(n-l)} T_{a(m-k)} g\right\rangle \\
& =\sum_{x, y \in \mathbb{Z}} h_{x, y}\left\langle v, M_{b(n-l-y)} T_{a(m-k-x)} s\right\rangle \omega^{-(m-k-x) y} \\
& =\sum_{x, y \in \mathbb{Z}} h_{x, y} r_{s v}[m-k-x, n-l-y] \omega^{-(m-k-x) y} \\
& =\left(h \bigsqcup r_{s v}\right)[m-k, n-l]=\delta_{m-k} \delta_{n-l},
\end{aligned}
$$

which completes the proof.

\section{F. Minimax Regret Synthesis}

Next, we develop a minimax-regret reconstruction method for noninvertible Gabor transforms with rational undersampling. The signal minimizing (23) can be obtained by applying $\mathcal{H}_{\mathrm{mx}}$ of (24) on the Gabor coefficients $c_{k, l}$ prior to synthesis. However, in contrast to the integer undersampling case discussed in Section V-B, where $V^{*} V, S^{*} V$, and $S^{*} S$ were convolution operators, here they correspond to twisted convolutions with $r_{v v}[k, l], r_{s v}[k, l]$, and $r_{s s}[k, l]$, respectively. Therefore, to obtain the sequence $d_{k, l}$, we apply a twisted convolution filter on the Gabor coefficients $c_{k, l}$, whose impulse response is

$$
h_{\mathrm{mx}}[k, l]=\left(r _ { v v } ^ { - 1 } \left\llcornerr_{s v}\left\llcorner r_{s s}^{-1}\right)[k, l] .\right.\right.
$$

Here, $r_{v v}^{-1}[k, l]$ and $r_{s s}^{-1}[k, l]$ are the inverses of $r_{v v}[k, l]$ and $r_{s s}[k, l]$ with respect to $\downarrow$. Consequently, the $\Phi$ function of the minimax-regret filter is given by

$$
\boldsymbol{H}_{\mathrm{mx}}(\omega, x)=\boldsymbol{\Phi}^{s s}(\omega, x)^{-1} \boldsymbol{\Phi}^{s v}(\omega, x) \underline{\boldsymbol{\Phi}}^{v v}(\omega, x)^{-1}
$$

where $\boldsymbol{\Phi}^{s s}(\omega, x), \boldsymbol{\Phi}^{s v}(\omega, x)$, and $\boldsymbol{\Phi}^{v v}(\omega, x)$ are the $\boldsymbol{\Phi}$-representations of $r_{s s}[k, l], r_{s v}[k, l]$, and $r_{v v}[k, l]$, respectively.

\section{G. Extension to Symplectic Lattices}

Throughout the current and previous sections, we considered a special type of sampling points in the time-frequency plane, called separable lattices $\Lambda=a \mathbb{Z} \times b \mathbb{Z}$. However, with the help of metaplectic operators, these results carry over to the more general class of lattices, called symplectic lattices. A lattice $\Lambda_{s} \subseteq \mathbb{R}^{2}$ is called symplectic, if one can write $\Lambda_{s}=\mathcal{D} \Lambda$ where $\Lambda$ is a separable lattice and $\mathcal{D} \in S L_{2}(\mathbb{R})$, meaning it is an invertible $2 \times 2$ matrix with determinant 1 [26]. To every $\mathcal{D} \in S L_{2}(\mathbb{R})$ there corresponds a unitary operator $\mu(\mathcal{D})$, called metaplectic operator, acting on $L^{2}(\mathbb{R})$. One can show that a Gabor system on a symplectic lattice is unitarily equivalent to a Gabor system on a separable lattice under $\mu(\mathcal{D})$. That is, $\mathcal{G}\left(g, \Lambda_{s}\right)$ is a frame/Riesz basis if and only if $\mathcal{G}\left(\mu(\mathcal{D})^{-1} g, \Lambda\right)$ is a frame/Riesz basis, and

$$
\mathcal{G}\left(g, \Lambda_{s}\right)=\mu(\mathcal{D}) \mathcal{G}\left(\mu(\mathcal{D})^{-1} g, \Lambda\right) \text {. }
$$

Therefore, instead of considering a representation of $f(t)$ in $\overline{\operatorname{span}}\left\{g_{\lambda}\right\}_{\lambda \in \Lambda_{s}}$ one can look at the representation of $f(t)$ in $\overline{\operatorname{span}}\left\{\mu(\mathcal{D})^{-1} g_{\lambda}\right\}_{\lambda \in \Lambda}$. For more details, see [26].

\section{SUBSPACE-PRIOR SYNTHESIS}

In the previous two sections we attempted to recover a signal from its noninvertible Gabor representation without using any prior knowledge on the signal. When such knowledge is available, it can significantly reduce the reconstruction error and in some cases even lead to perfect recovery. A common prior in sampling theory is that the signal to be recovered lies in some SI subspace of $L^{2}$ with a single generator. Namely that it can be written as

$$
f(t)=\sum_{k \in \mathbb{Z}} d_{k} T_{a k} w(t)=\sum_{k \in \mathbb{Z}} d_{k} w(t-a k)
$$

with a norm-bounded sequence $\left\{d_{k}\right\}$ and some window $w(t)$. This model can quite accurately describe many types of natural signals, which exhibit a certain degree of smoothness. For example, the class of bandlimited signals is the SI space generated by the sinc window. The class of splines of degree $N$ also follows this description with $w(t)$ being the B-spline function of degree $N$.

Here, we generalize the SI-prior setting to SMI spaces. We use these spaces as priors on our input signals, in order to recover them from their noninvertible Gabor transform. Our setting is thus as follows. Specifically, we assume that $f(t)$ lies in some SMI space $\mathcal{W}$, generated by $\mathcal{G}(w, a, b)$, which we term the prior space. That means that $f(t)$ can be represented in the form

$$
f(t)=\sum_{k, l \in \mathbb{Z}} h_{k, l} M_{b l} T_{a k} w(t)
$$

for some sequence $h_{k, l}$ in $\ell^{2}\left(\mathbb{Z}^{2}\right)$. We are given the Gabor coefficients $c_{k, l}$ of $f(t)$, which were computed with the analysis window $s(t)$. Our goal is to produce a recovery $\tilde{f}(t)$ using the synthesis window $v(t)$. Clearly, if $\mathcal{W}$ does not coincide with our synthesis space $\mathcal{V}$, then the reconstruction $\tilde{f}(t)$ cannot equal $f(t)$. The interesting question is whether we can obtain the best possible recovery, which is the orthogonal projection $\tilde{f}=P_{\mathcal{V}} f$, from the Gabor coefficients $c_{k, l}$ of $f(t)$. In our developments, we discuss the integer and rational undersampling cases separately.

\section{A. Integer Undersampling}

As discussed in Section IV-C, if the analysis and prior spaces satisfy $\mathcal{S}^{\perp} \oplus \mathcal{W}=L^{2}(\mathbb{R})$, then the best possible recovery can be generated by applying the operator $\mathcal{H}_{\text {sub }}(25)$ on the Gabor coefficients $c_{k, l}$ prior to synthesis. From Proposition V.1 we know that this direct-sum condition is satisfied if and only if $\Phi^{s w}(\omega, x) \neq 0$ almost everywhere, where $\Phi^{s w}(\omega, x)$ is as in (26) with $v(t)$ replaced by $w(t)$. The operators $V^{*} V, V^{*} W$, and $S^{*} W$ correspond to 2D convolutions with the kernels $r_{v v}[k, l]$, $r_{v w}[k, l]$ and $r_{w w}[k, l]$, respectively, which are given by (27) with the appropriate substitution of $s(t), v(t)$ and $w(t)$. Therefore, $\mathcal{H}_{\text {sub }}$ corresponds to $2 \mathrm{D}$ convolution with the filter $h_{\text {sub }}$, whose 2D DTFT is given by

$$
H_{\mathrm{sub}}(\omega, x)=\frac{\Phi^{v w}(\omega, x)}{\Phi^{s w}(\omega, x) \Phi^{v v}(\omega, x)}
$$


where $\Phi^{v w}(\omega, x), \Phi^{s w}(\omega, x)$, and $\Phi^{v v}(\omega, x)$ are the 2D DTFTs of $r_{v w}[k, l], r_{s w}[k, l]$ and $r_{v v}[k, l]$, respectively.

When the synthesis space $\mathcal{V}$ coincides with the prior space $\mathcal{W}$, we have $\mathcal{H}_{\text {sub }}=\left(V^{*} V\right)^{-1} V^{*} W\left(S^{*} W\right)^{-1}=\left(S^{*} W\right)^{-1}$, so that the correction filter is the same as in the consistency approach of Section V-A. In this case, the direct-sum condition (namely the invertibility of the operator $S^{*} W$ ) guarantees perfect recovery of $f(t)$. To see this, note that any $f \in \mathcal{W}$ can be written as $f=W d$ for some sequence $d_{k, l}$, so that the Gabor coefficients $c_{k, l}$ are given by $c=S^{*} f=S^{*} W d$. Therefore, the expansion coefficients can be perfectly recovered using $d=\left(S^{*} W\right)^{-1} c$. This property is, of course, independent of the sampling lattice and holds true also in the rational undersampling regime.

\section{B. Rational Undersampling}

We now extend the subspace-prior approach to the rational undersampling regime. As before, we assume that the input $f(t)$ can be expressed in the form (39) for some sequence $h_{k, l}$. The best possible recovery can be obtained by applying the operator $\mathcal{H}_{\text {sub }}$ of (25) on the Gabor transform $c_{k, l}$ prior to reconstruction. The operators $V^{*} V, V^{*} W$, and $S^{*} W$ correspond to twisted convolution with the kernels $r_{v v}[k, l], r_{v, w}[k, l]$, and $r_{s, w}[k, l]$, respectively. Therefore, $\mathcal{H}_{\text {sub }}$ corresponds to twisted convolution with

$$
h_{\mathrm{sub}}[k, l]=\left(r _ { v v } ^ { - 1 } \left\llcornerr_{v w}\left\llcorner r_{s w}^{-1}\right)[k, l]\right.\right.
$$

The corresponding $\boldsymbol{\Phi}$ function is given by

$$
\boldsymbol{H}_{\mathrm{sub}}(\omega, x)=\boldsymbol{\Phi}^{s w}(\omega, x)^{-1} \boldsymbol{\Phi}^{v w}(\omega, x) \boldsymbol{\Phi}^{v v}(\omega, x)^{-1}
$$

where $\boldsymbol{\Phi}^{s w}(\omega, x), \boldsymbol{\Phi}^{v w}(\omega, x)$, and $\boldsymbol{\Phi}^{v v}(\omega, x)$ are the $\boldsymbol{\Phi}$-representations of $r_{s w}[k, l], r_{v w}[k, l]$, and $r_{v v}[k, l]$, respectively.

\section{EXAMPLE: INTEGER UNDERSAMPLING WITH GAUSSIAN WINDOWS}

We now demonstrate the prior-free recovery techniques derived in this paper. To retain simplicity we will focus on the integer undersampling scenario. In this regime, the smallest amount of information loss occurs when $a b=2$. Therefore, in our simulations we used $a=1$ and $b=2$. In this setting there are at most half the number of time-frequency coefficients for any given frequency range per time unit, than in any invertible Gabor representation. Consequently, algorithms operating in the Gabor domain (e.g., for system identification, speech enhancement, blind source separation, etc.) will benefit from a reduction of at least a factor of 2 in computational load. On the other hand, we expect the norm of the reconstruction error to be roughly on the order of the signal's norm in the worst-case scenario, since half of the information is lost in such a representation.

For tractability, we consider the case in which the analysis and synthesis are both performed with Gaussian windows:

$$
\begin{aligned}
& s(t)=\frac{1}{\sqrt{2 \pi \sigma_{s}^{2}}} \exp \left\{-\frac{t^{2}}{2 \sigma_{s}^{2}}\right\} \\
& v(t)=\frac{1}{\sqrt{2 \pi \sigma_{v}^{2}}} \exp \left\{-\frac{t^{2}}{2 \sigma_{v}^{2}}\right\} .
\end{aligned}
$$
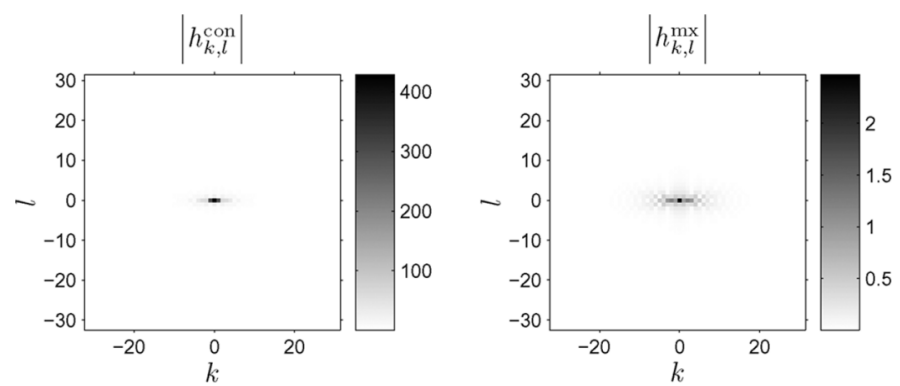

Fig. 3. The $2 \mathrm{D}$ correction filters corresponding to the minimax-regret and consistency methods.
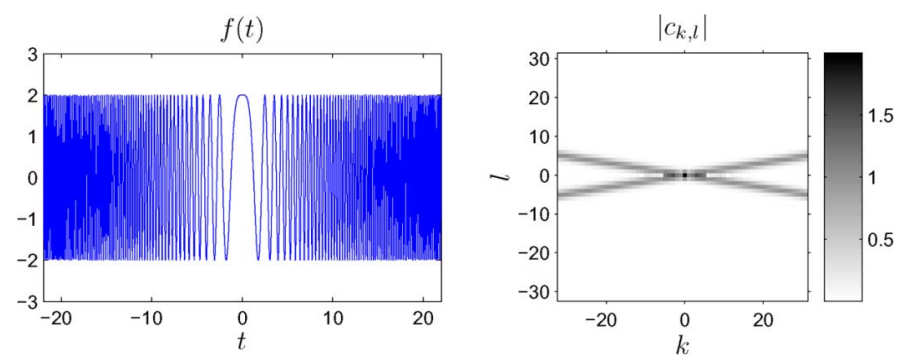

Fig. 4. A chirp signal and its Gaussian-window Gabor representation.

In this scenario, the cross-correlation sequence $r_{s v}[k, l]=$ $\left\langle v, M_{b n} T_{a m} s\right\rangle$, has an analytic expression:

$$
\begin{aligned}
r_{s v}[k, l]=\frac{1}{\sqrt{2 \pi\left(\sigma_{s}^{2}+\sigma_{v}^{2}\right)}} \exp \{ & \left.-\frac{(a k)^{2}+4 \pi^{2} \sigma_{s}^{2} \sigma_{v}^{2}(b l)^{2}}{q 2\left(\sigma_{s}^{2}+\sigma_{v}^{2}\right)}\right\} \\
\cdot & \exp \left\{\frac{2 \pi i \sigma_{s}^{2} a b k l}{\sigma_{s}^{2}+\sigma_{v}^{2}}\right\} .
\end{aligned}
$$

Similarly, $r_{s s}[k, l]$ and $r_{v v}[k, l]$ can be obtained by replacing $\sigma_{s}$ by $\sigma_{v}$ and vice versa.

The $2 D$ filter $h_{\text {con }}$ of (32), corresponding to the consistency requirement, is the convolutional inverse of $r_{s s}[k, l]$. This sequence can be approximated numerically using the discrete Fourier transform (DFT) of the finite-length sequence $r_{s v}[k, l]$, $(k, l) \in[-K, K] \times[-L, L]$, for some (large) $K$ and $L$. To compute the filter $h_{\mathrm{mx}}$ of (35), corresponding to the minimax-regret approach, we need to invert $r_{s s}[k, l]$ and $r_{v v}[k, l]$, which can be done in a similar manner. Note that both $h_{\text {con }}$ and $h_{\mathrm{mx}}$ are generally complex sequences. Fig. 3 depicts the modulus $\left|h_{\text {con }}\right|$ and $\left|h_{\mathrm{mx}}\right|$ for the case $\sigma_{s}=0.1, \sigma_{v}=2$, and $a b=2$.

To see the effect of these two filters, we now examine the recovery of a chirp signal from its noninvertible Gabor representation using both methods. Specifically, let

$$
f(t)=2 \cos \left(t^{2}\right) .
$$

The Gaussian-window Gabor transform of $f(t)$ has a closed form expression, given by

$$
\begin{aligned}
c_{k, l}= & \frac{1}{\sqrt{-2 i \sigma_{s}^{2}+1}} \exp \left\{-\frac{a k(a k+2 b l \pi)+2 i b^{2} l^{2} \pi^{2} \sigma_{s}^{2}}{i+2 \sigma_{s}^{2}}\right\} \\
& +\frac{1}{\sqrt{2 i \sigma_{s}^{2}+1}} \exp \left\{\frac{-a k(a k-2 b l \pi)+2 i b^{2} l^{2} \pi^{2} \sigma_{s}^{2}}{-i+2 \sigma_{s}^{2}}\right\} .
\end{aligned}
$$

The signal $f(t)$ and the modulus of its Gabor transform, $\left|c_{k, l}\right|$, are shown in Fig. 4. Although $c_{k, l}$ seems to constitute a good time-frequency representation of $f(t)$, it is certainly not 


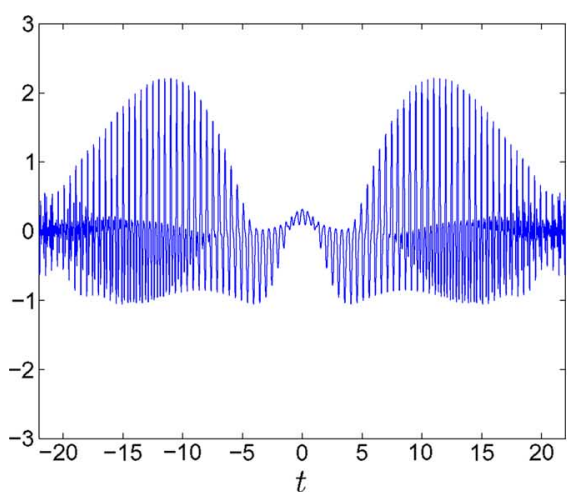

(a)

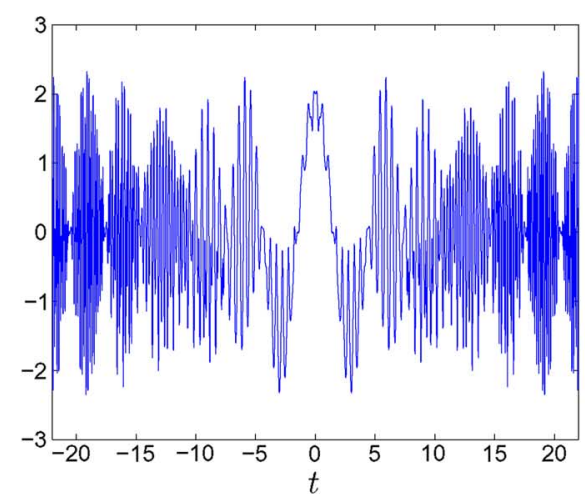

(b)

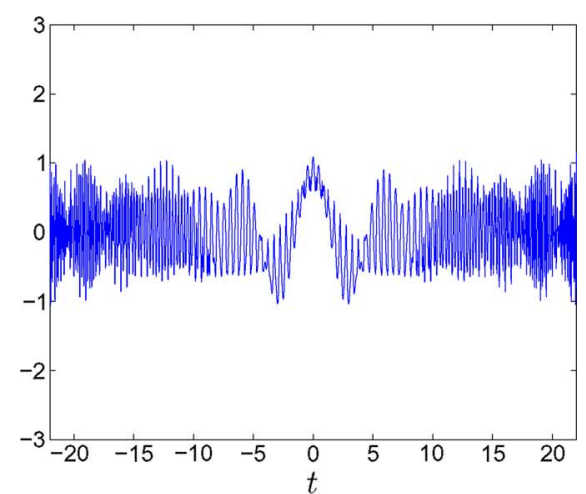

(c)

Fig. 5. Reconstructions of $f(t)$ from its Gabor coefficients $c_{k, l}$. (a) Without processing $c_{k, l}$. (b) Consistent recovery, namely using $d_{k, l}=\left(c * h_{\text {con }}\right)_{k, l}$ as expansion coefficients. (c) Minimax-regret recovery, corresponding to $d_{k, l}=\left(c * h_{\mathrm{mx}}\right)_{k, l}$.
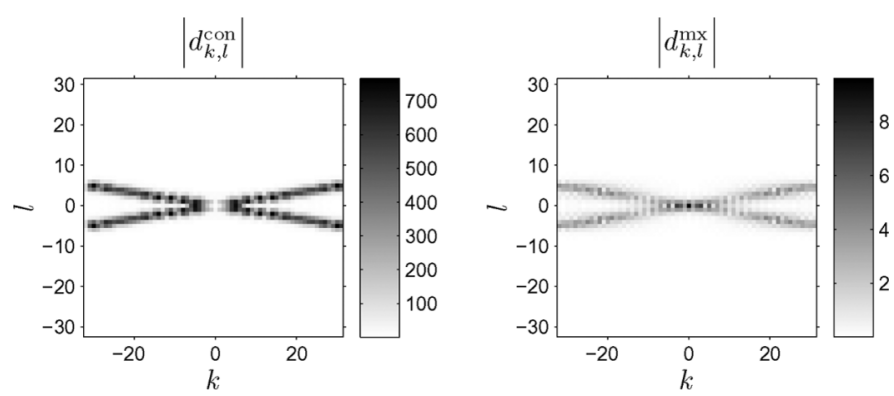

Fig. 6. The modulus of the expansion coefficients $\left|d_{k, l}\right|$ corresponding to the consistent and minimax-regret recovery methods.

suited to play the role of the synthesis expansion coefficients $d_{k, l}$. This can be seen in Fig. 5(a), where $c_{k, l}$ have been used without modification as expansion coefficients to produce a recovery $\tilde{f}(t)$. The signal-to-noise ratio (SNR) of this recovery is $20 \log _{10}(\|f\| /\|f-\tilde{f}\|)=-0.44 \mathrm{~dB}$.

The reconstructions obtained with the consistency and minimax-regret methods are shown in Fig. 5(b) and (c). Clearly, they both bear better resemblance to $f(t)$. The consistent recovery is the unique signal that can be constructed with the synthesis window $v(t)$, whose Gabor transform coincides with $c_{k, l}$. This property makes this reconstruction desirable in some sense, although its SNR is only $-1.03 \mathrm{~dB}$, worse than the uncompensated recovery. To guarantee that the error between our recovery $\tilde{f}(t)$ and the original signal $f(t)$ is small, for every possible $f(t)$ that could have generated $c_{k, l}$, one has to use the minimax regret approach, as shown Fig. 5(c). This reconstruction achieves an SNR of $0.1 \mathrm{~dB}$, and therefore is better than the other two methods in terms of reconstruction error. Fig. 6 depicts the expansion coefficients $d_{k, l}$ corresponding to the two methods.

\section{CONCLUSION}

In this paper, we explored various techniques for recovering a signal from its noninvertible Gabor transform, where the undersampling factor is rational. Specifically, we studied situations where both the analysis and synthesis windows of the transform are given, so that the only freedom is in processing the coefficients in the time-frequency domain prior to synthesis. We began with the consistency approach, in which the recovered signal is required to possess the same Gabor transform as the original signal. We then analyzed a minimax strategy whereby a reconstruction with minimal worst case error is sought. Finally, we developed a recovery method yielding the minimal possible error when the original signal is known to lie in some given Gabor space. We showed that all three techniques amount to performing a 2D twisted convolution operation on the Gabor coefficients prior to synthesis. When the undersampling factor of the transform is an integer, this process reduces to standard 2D convolution. We demonstrated our techniques for Gaussianwindow transforms in the context of recovering a chirp signal.

\section{APPENDIX A \\ Twisted CONVOLUTION}

In Section VI, we saw that in order to process the samples $c_{m, n}$ one needs the inverse of certain cross-correlation sequences with respect to $\measuredangle$. In this section we show how to obtain explicitly the inverse of a sequence $d_{k, l}$ with respect to twisted convolution with parameter $a b$. This depends very much on $a b$. If $a b \in \mathbb{N}$, then the twisted convolution is a standard convolution, and the Fourier transform can be used to compute the inverse of $d_{k, l}$. If $a b=q / p$, then one can use the construction derived in [25], which breaks the problem into computing inverses of several sequences with respect to standard convolution. We now briefly review this method. For the proofs and more detailed explanations, we refer the reader to the original paper.

Let $d_{k, l}$ be a sequence in $\ell^{1}\left(\mathbb{Z}^{2}\right)$. We create $p^{2}$ new sequences out of $d_{k, l}$, defined as

$$
\left(d^{r, s}\right)_{k, l}=d_{k, l} \sum_{m \in \mathbb{Z}} \sum_{n \in \mathbb{Z}} \delta[k-r-p m, l-s-p n]
$$

where $r, s=0,1, \ldots, p-1$. It is easy to see that the sequence $d^{r, s}$ is supported on the coset $(r+p \mathbb{Z}) \times(s+p \mathbb{Z})$ and therefore $d=\sum_{r=0}^{p-1} \sum_{s=0}^{p-1} d^{r, s}$. In the case in which $p=2$, out of a sequence $d_{k, l}$ we obtain four subsequences: $d^{0,0}$ which is supported on $2 \mathbb{Z} \times 2 \mathbb{Z}, d^{0,1}$ supported on $2 \mathbb{Z} \times(2 \mathbb{Z}+1), d^{1,0}$ supported on $(2 \mathbb{Z}+1) \times 2 \mathbb{Z}$ and $d^{1,1}$ supported on $(2 \mathbb{Z}+1) \times(2 \mathbb{Z}+1)$.

Next, we associate with the sequence $d_{k, l}$ a $p \times p$ matrix $D$ whose entries are sequences in $\ell^{1}$ :

$$
D_{r, s}=\sum_{m=0}^{p-1} d^{m, r-s} e^{-2 \pi i m s q / p}
$$


where $r-s$ should be interpreted as modulo $p$. This matrix is an element of an algebra $\mathcal{M}$ of $p \times p$ matrices with multiplication of two matrices $D$ and $E$ given by

$$
(D \circledast E)_{r, s}=\sum_{m=0}^{p-1} D_{r, l} * E_{l, s}
$$

where $*$ is a standard convolution. It was shown in [25] that an algebra of such matrices is closed under taking inverses, meaning that if $D$ is invertible in $\mathcal{M}$ then its inverse is also an element of $\mathcal{M}$ and its entries are also coming from some sequence in $\ell^{1}\left(\mathbb{Z}^{2}\right)$. For example, when $p=2$ the above matrix takes the form

$$
D=\left(\begin{array}{ll}
d^{0,0}+d^{1,0} & d^{0,1}-d^{1,1} \\
d^{0,1}+d^{1,1} & d^{0,0}-d^{1,0}
\end{array}\right)
$$

where we used the fact that since $p=2, q$ must be odd, and thus $e^{2 \pi i m s q / 2}$ for $m, s=0,1$ takes the values 1 and -1 . Note that summing up the elements of the first column gives us back the sequence $d$.

It was shown in [25] that the invertibility of the sequence $d_{k, l}$ with respect to $t$ is equivalent to the invertibility of the matrix $D$ in this new matrix algebra, which in turn is equivalent to the invertibility of $\operatorname{det}(D)$ in $\ell^{1}\left(\mathbb{Z}^{2}, *\right)$. Therefore, if $D$ is invertible, its inverse can be computed using Cramer's Rule. That is the $(r, s)$ entry of $D^{-1}$ is given by

$$
\left(D^{-1}\right)_{r, s}=(\operatorname{det}(D))^{-1} * \operatorname{det}(D(s, r))
$$

where $D(s, r)$ is a $p \times p$ matrix obtained from $D$ by substituting the $s$ th row of $D$ with a vector of zeros having $\delta$ on the $r$ th position, and the $r$ th column with a column of zeros having $\delta$ on the $s$ th position. Note that $\operatorname{det}(D)$ is a sequence and its inverse in (45) is taken with respect to standard convolution. For example, when $p=2$ we get

$$
\begin{aligned}
& D(0,0)=\left(\begin{array}{ccc}
\delta & 0 \\
0 & d^{0,0}-d^{1,0}
\end{array}\right) \\
& D(1,0)=\left(\begin{array}{cc}
0 & \delta \\
d^{0,1}+d^{1,1} & 0
\end{array}\right) \\
& D(0,1)=\left(\begin{array}{cc}
0 & d^{0,1}-d^{1,1} \\
\delta & 0
\end{array}\right) \\
& D(1,1)=\left(\begin{array}{cc}
d^{0,0}+d^{1,0} & 0 \\
0 & \delta
\end{array}\right) .
\end{aligned}
$$

Thus

$$
D^{-1}=(\operatorname{det} D)^{-1} *\left(\begin{array}{cc}
d^{0,0}-d^{1,0} & -d^{0,1}+d^{1,1} \\
-d^{0,1}-d^{1,1} & d^{0,0}+d^{1,0}
\end{array}\right)
$$

where $\operatorname{det}(D)=\left(d^{0,0}+d^{1,0}\right) *\left(d^{0,0}-d^{1,0}\right)-\left(d^{0,1}+\right.$ $\left.d^{1,1}\right) *\left(d^{0,1}-d^{1,1}\right)$. Since the matrix algebra $\mathcal{M}$ is closed under taking inverses, summing up the elements of the first column of $D^{-1}$ results in some sequence $e_{k, l}$ which is the inverse of $d_{k, l}$ with respect to twisted convolution. Therefore, it is enough to compute only this column and sum up its entries to get $d^{-1}$. In our example with $p=2$, the twisted-convolutional-inverse of $d$ equals $(\operatorname{det}(D))^{-1} *\left(d^{0,0}-d^{1,0}-d^{0,1}-d^{1,1}\right)$.

We mentioned in the previous sections that it is possible to realize twisted convolution with a rational parameter $a b$ using a filter bank. Indeed, using the decomposition (43), the twisted convolution of two sequences $c$ and $d$,

$$
\begin{aligned}
(d \curvearrowleft c)_{m, n} & =\sum_{k, l \in \mathbb{Z}} d_{k, l} c_{m-k, n-l} e^{-2 \pi i a b(m-k) l} \\
& =\sum_{k, l \in \mathbb{Z}} c_{k, l} d_{m-k, n-l} e^{-2 \pi i a b(n-l) k}
\end{aligned}
$$

can be written as

$$
(d \llbracket c)=\sum_{r, s=0}^{p-1} \sum_{u, v=0}^{p-1}\left(c^{r, s} * d^{u-r, v-s}\right) e^{-2 \pi i(v-s) r q / p}
$$

for $u, v=0,1, \ldots, p-1$. Therefore, as shown in Fig. 7, each of the $p^{2}$ sequences $c^{r, s}, r, s=0,1, \ldots, p-1$, is split into $p^{2}$ filters associated with the sequences $d^{u, v}, u, v=0,1, \ldots, p-1$. Then, $d \llbracket c$ is obtained by summing over the resulting $p^{4}$ output sequences. Fig. 7 depicts one of the $p^{4}$ branches, which corresponds to the indices $r, s, u$ and $v$.

\section{APPENDIX B \\ The Multiplication Property of $\boldsymbol{\Phi}$}

In this appendix we prove the multiplication property of $\boldsymbol{\Phi}$. Let $c_{k, l}$ and $d_{k, l}$ be two sequences having $\boldsymbol{\Phi}$ matrix-valued function representations $\boldsymbol{\Phi}^{c}$ and $\boldsymbol{\Phi}^{d}$, respectively. Then the matrixvalued function $\Phi$ associated with the twisted convolution $c \curvearrowleft d$, can be expressed as

$$
\boldsymbol{\Phi}^{(c \natural d)}(\omega, x)=\boldsymbol{\Phi}^{d}(\omega, x) \boldsymbol{\Phi}^{c}(\omega, x) .
$$

To show this, let $a b=q / p$ and let $r, s=0, \ldots, p-1$ be fixed. Then

$$
\begin{aligned}
\boldsymbol{\Phi}_{r, s}^{(c \bigsqcup d)}(\omega, x)= & \sum_{k, l \in \mathbb{Z}}(c \bigsqcup d)[s-r+p k, l] e^{-2 \pi i a b r l} \\
& \cdot e^{-2 \pi i(b l x+a k \omega)} \\
= & \sum_{k, l \in \mathbb{Z}} \sum_{m, n \in \mathbb{Z}} c_{m, n} d_{s-r+p k-m, l-n} \\
& \cdot e^{-2 \pi i a b(s-r+p k-m) n} \\
& \cdot e^{-2 \pi i a b r l} e^{-2 \pi i(b l x+a k \omega)} .
\end{aligned}
$$

Using the decomposition (43) of the sequences, (47) becomes

$$
\begin{aligned}
\boldsymbol{\Phi}_{r, s}^{(c \Downarrow d)}(\omega, x)= & \sum_{u=0}^{p-1} \sum_{k, l \in \mathbb{Z}} \sum_{m, n \in \mathbb{Z}} c_{u+p m, n} d_{s-r-u+p(k-m), l-n} \\
& \cdot e^{-2 \pi i a b(s-r-u) n} e^{-2 \pi i a b r l} e^{-2 \pi i(b l x+a k \omega)} \\
= & \sum_{u=0}^{p-1} \sum_{k, l \in \mathbb{Z}} \sum_{m, n \in \mathbb{Z}} c_{s-u+p m, n} d_{u-r+p k, l} \\
& \cdot e^{-2 \pi i a b(u-r) n} e^{-2 \pi i a b r(l+n)} \\
& \cdot e^{-2 \pi i(b(l+n) x+a(k+m) \omega)}
\end{aligned}
$$




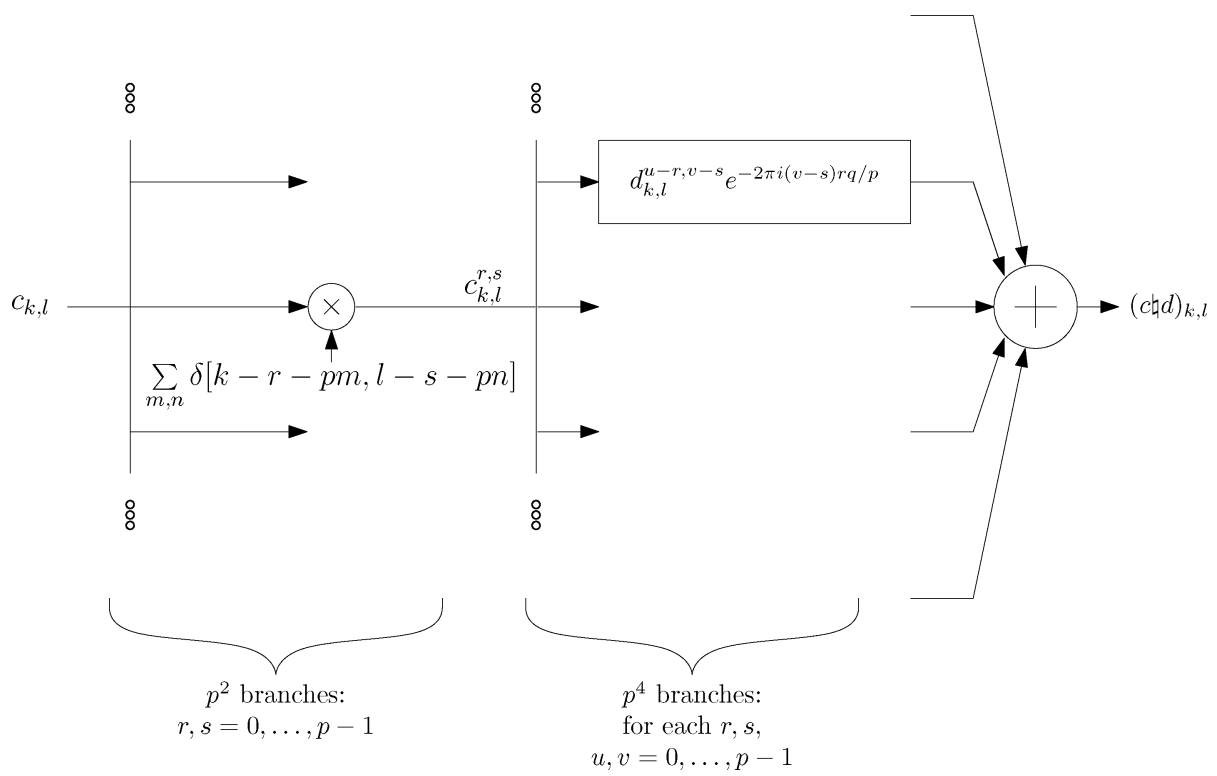

Fig. 7. A filter-bank realization of twisted convolution between $c_{k, l}$ and $d_{k, l}$.

Rearranging the sums in the above equation we arrive at

$$
\begin{aligned}
\boldsymbol{\Phi}_{r, s}^{(c \bigsqcup d)}(\omega, x) & \sum_{u=0}^{p-1}\left(\sum_{k, l \in \mathbb{Z}} d_{u-r+p k, l} e^{-2 \pi i a b r l} e^{-2 \pi i(\text { blx }+a k \omega)}\right) \\
& \cdot\left(\sum_{m, n \in \mathbb{Z}} c_{s-u+p m, n} e^{-2 \pi i a b u n} e^{-2 \pi i(b n x+a m \omega)}\right) \\
= & \sum_{u=0}^{p-1} \boldsymbol{\Phi}_{r, u}^{d}(\omega, x) \boldsymbol{\Phi}_{u, s}^{c}(\omega, x) .
\end{aligned}
$$

Hence, $\boldsymbol{\Phi}^{(c \bigsqcup d)}(\omega, x)=\boldsymbol{\Phi}^{d}(\omega, x) \boldsymbol{\Phi}^{c}(\omega, x)$.

\section{APPENDIX C}

\section{PROOF OF PROPOSITION III.3}

Since $\mathcal{G}(v, a, b)$ is a Riesz basis for $\mathcal{V}$, there exist bounds $A>0$ and $B<\infty$ such that $A \boldsymbol{I}_{p} \leq \boldsymbol{\Phi}^{v v}(\omega, x) \leq B \boldsymbol{I}_{p}$, where $\boldsymbol{\Phi}^{v v}(\omega, x)$ is the matrix-valued function associated with the sequence $r_{v v}[k, l]$, defined in (11). The system $\mathcal{G}(w, a, b)$, with $w(t)=\sum_{k, l \in \mathbb{Z}} h_{k, l} M_{b l} T_{a k} v(t)$, is a Riesz basis if and only if there exist constants $C>0$ and $D<\infty$ such that

$$
C \underline{\boldsymbol{I}}_{p} \leq \boldsymbol{\Phi}^{w w}(\omega, x) \leq D \underline{\boldsymbol{I}}_{p}
$$

Here $\Phi^{w}(\omega, x)$ is a matrix-valued function built from the crosscorrelation sequence $r_{w w}[k, l]=\left\langle w, M_{b l} T_{a k} w\right\rangle$. By substituting $w(t)=\sum_{k, l \in \mathbb{Z}} h_{k, l} M_{b l} T_{a k} v(t)$ in $r_{w w}[k, l]$ one obtains

$$
\begin{aligned}
r_{w w}[k, l]= & \sum_{y, z \in \mathbb{Z}} \sum_{m, n \in \mathbb{Z}} r_{v v}[y-m, z-n] h_{m, n} \\
& \cdot e^{-2 \pi i a b(z-n) m} \overline{h_{y-k, z-l}} e^{2 \pi i a b(z-l) k} \\
= & \sum_{y, z \in \mathbb{Z}}\left(r_{v v} \llbracket h\right)[y, z] \overline{h_{y-k, z-l}} e^{2 \pi i a b(z-l) k} \\
= & \left(h^{*} \llbracket r_{v v} \llbracket h\right)[k, l],
\end{aligned}
$$

where $r_{v v}[m, n]=\left\langle v, M_{b n} T_{a m} v\right\rangle$ and $h^{*}[k, l]=\overline{h_{-k,-l}}$. It is easy to check, and we leave it for the reader, that $\Phi^{h^{*}}(\omega, x)=$ $\Phi^{h}(\omega, x)^{H}$. Therefore, using the relation from Appendix B, the $(r, s)$-entry of the matrix $\boldsymbol{\Phi}^{w w}(\omega, x)$ is

$$
\boldsymbol{\Phi}_{r, s}^{w w}(\omega, x)=\left(\boldsymbol{\Phi}^{h}(\omega, x) \boldsymbol{\Phi}^{v v}(\omega, x) \boldsymbol{\Phi}^{h}(\omega, x)^{H}\right)_{r, s}
$$

where $\boldsymbol{\Phi}^{h}(\omega, x)$ is a matrix-valued function associated with the sequence $h_{k, l}$ and defined in the Proposition. Hence, if $\mathcal{G}(w, a, b)$ and $\mathcal{G}(v, a, b)$ are Riesz bases with bounds $C>0$, $D<\infty$, and $A>0, B<\infty$, respectively, then

$$
\begin{aligned}
\boldsymbol{\Phi}^{h}(\omega, x) \boldsymbol{\Phi}^{h}(\omega, x)^{H} & \geq \frac{1}{A} \boldsymbol{\Phi}^{h}(\omega, x) \boldsymbol{\Phi}^{v v}(\omega, x) \boldsymbol{\Phi}^{h}(\omega, x)^{H} \\
& =\frac{1}{A} \boldsymbol{\Phi}^{w w}(\omega, x) \geq \frac{D}{A} \\
\boldsymbol{\Phi}^{h}(\omega, x) \boldsymbol{\Phi}^{h}(\omega, x)^{H} & \leq \frac{1}{B} \boldsymbol{\Phi}^{h}(\omega, x) \boldsymbol{\Phi}^{v v}(\omega, x) \boldsymbol{\Phi}^{h}(\omega, x)^{H} \\
& =\frac{1}{B} \boldsymbol{\Phi}^{w w}(\omega, x) \leq \frac{C}{B} .
\end{aligned}
$$

Therefore $\Phi^{h}(\omega, x)$ satisfies (16) with bounds $m=C / B$ and $M=D / A$.

On the other hand, if the sequence $h_{k, l}$ is such that (16) is satisfied, then

$$
\begin{aligned}
\boldsymbol{\Phi}^{w w}(\omega, x) & =\boldsymbol{\Phi}^{h}(\omega, x) \boldsymbol{\Phi}^{v v}(\omega, x) \boldsymbol{\Phi}^{h}(\omega, x)^{H} \\
& \geq A \boldsymbol{\Phi}^{h}(\omega, x) \boldsymbol{\Phi}^{h}(\omega, x)^{H} \geq A m \\
\boldsymbol{\Phi}^{w w}(\omega, x) & =\boldsymbol{\Phi}^{h}(\omega, x) \boldsymbol{\Phi}^{v v}(\omega, x) \boldsymbol{\Phi}^{h}(\omega, x)^{H} \\
& \leq B \boldsymbol{\Phi}^{h}(\omega, x) \boldsymbol{\Phi}^{h}(\omega, x)^{H} \leq B M,
\end{aligned}
$$

and so $\mathcal{G}(w, a, b)$ is a Riesz basis with bounds $C=A m$ and $D=B M$. It remains to show that $\mathcal{G}(w, a, b)$ and $\mathcal{G}(v, a, b)$ span the same space. Every element of $\mathcal{G}(w, a, b)$ can be uniquely represented by a linear combinations of the elements from $\mathcal{G}(v, a, b)$, since the latter is a Riesz basis. It suffices to show that $v(t)$ can be written as a linear combination of the elements from $\mathcal{G}(w, a, b)$ (it will be a unique representation 
since $\mathcal{G}(w, a, b)$ is a Riesz basis). Then, since Gabor spaces are closed under translation and modulations, other basis elements from $\mathcal{G}(v, a, b)$ will also admit a unique representation in terms of $\mathcal{G}(w, a, b)$. Let $g_{k, l}$ be the inverse of $h_{k, l}$ with respect to $h$, meaning $h \bigsqcup g=\delta$. The inverse exists because $h_{k, l}$ satisfies (16). Let $\tilde{v}(t)=\sum_{m, n \in \mathbb{Z}} g_{m, n} M_{b n} T_{a m} w(t)$. We will now show that $\tilde{v}(t)=v(t)$. Indeed

$$
\begin{aligned}
\tilde{v}(t) & =\sum_{m, n \in \mathbb{Z}} g_{m, n} M_{b n} T_{a m} w(t) \\
& =\sum_{m, n \in \mathbb{Z}} \sum_{k, l \in \mathbb{Z}} g_{m, n} h_{k, l} M_{b n} T_{a m} M_{b l} T_{a k} v(t) \\
& =\sum_{m, n \in \mathbb{Z}} g_{m, n} \sum_{k, l \in \mathbb{Z}} h_{k, l} e^{-2 \pi i a b m l} M_{b(n+l)} T_{a(m+k)} v(t) \\
& =\sum_{m, n \in \mathbb{Z}} \sum_{k, l \in \mathbb{Z}} g_{m-k, n-l} h_{k, l} e^{-2 \pi i a b(m-k) l} M_{b n} T_{a m} v(t) \\
& =\sum_{m, n \in \mathbb{Z}}(h \llbracket g)[m, n] M_{b n} T_{a m} v(t)=v(t) .
\end{aligned}
$$

\section{REFERENCES}

[1] Y. Ephraim and D. Malah, "Speech enhancement using a minimum-mean square error short-time spectral amplitude estimator," IEEE Trans. Acoust. Speech Signal Process., vol. 32, no. 6, pp. 1109-1121, 1984.

[2] I. Cohen and B. Berdugo, "Speech enhancement for nonstationary noise environments," Signal Process., vol. 81, no. 11, pp. 2403-2418, 2001.

[3] A. Belouchrani and M. G. Amin, "Blind source separation based on time-frequency signal representations," IEEE Trans. Signal Process., vol. 46, no. 11, pp. 2888-2897, 1998.

[4] Y. Lu and J. M. Morris, "Gabor expansion for adaptive echo cancellation," IEEE Signal Process. Mag., vol. 16, no. 2, pp. 68-80, 1999.

[5] C. Avendano, C. A. T. Center, and S. Valley, "Acoustic echo suppression in the STFT domain," in Proc. IEEE Workshop Appl. Signal Process. Audio Acoust., 2001, pp. 175-178.

[6] I. Cohen, "Relative transfer function identification using speech signals," IEEE Trans. Speech Audio Process., vol. 12, no. 5, pp. 451-459, 2004.

[7] S. Gannot, D. Burshtein, and E. Weinstein, "Signal enhancement using beamforming and nonstationarity withapplications to speech," IEEE Trans. Signal Process., vol. 49, no. 8, pp. 1614-1626, 2001.

[8] Y. Avargel and I. Cohen, "System identification in the short-time Fourier transform domain with crossband filtering," IEEE Trans. Audio Speech Lang. Process., vol. 15, no. 4, p. 1305, 2007.

[9] S. Tomazic and S. Znidar, "A fast recursive STFT algorithm," in Proc. 8th Medit. Electrotech. Conf., 1996 (MELECON'96), 1996, vol. 2.

[10] M. Unser, "Sampling—50 years after shannon," IEEE Proc., vol. 88, pp. 569-587, Apr. 2000.

[11] Y. C. Eldar and T. Michaeli, "Beyond bandlimited sampling," IEEE Signal Process. Mag., vol. 26, no. 3, pp. 46-68, May 2009.

[12] M. Unser and A. Aldroubi, "A general sampling theory for nonideal acquisition devices," IEEE Trans. Signal Process., vol. 42, no. 11, pp. 2915-2925, Nov. 1994.

[13] Y. C. Eldar and T. G. Dvorkind, "A minimum squared-error framework for generalized sampling," IEEE Trans. Signal Process., vol. 54, no. 6, pp. 2155-2167, Jun. 2006.

[14] M. Unser, A. Aldroubi, and M. Eden, "B-Spline signal processing: Part I-Theory," IEEE Trans. Signal Process., vol. 41, no. 2, pp. 821-833, Feb. 1993.

[15] A. Aldroubi and K. Gröchenig, "Non-uniform sampling and reconstruction in shift-invariant spaces," SIAM Rev., vol. 43, pp. 585-620, 2001.

[16] A. Aldroubi, "Non-uniform weighted average sampling and exact reconstruction in shift-invariant and wavelet spaces," Appl. Comp. Harmon. Anal., vol. 13, pp. 151-161, 2002.

[17] O. Christensen and Y. C. Eldar, "Oblique dual frames and shift-invariant spaces,” Appl. Computat. Harmon. Anal., vol. 17, no. 1, 2004.
[18] M. Unser and T. Blu, "Cardinal exponential splines: Part I-Theory and filtering algorithms," IEEE Trans. Signal Process., vol. 53, no. 4, pp. 1425-1438, Apr. 2005.

[19] M. Unser and T. Blu, "Generalized smoothing splines and the optimal discretization of the Wiener filter," IEEE Trans. Signal Process., vol. 53, no. 6, pp. 2146-2159, 2005.

[20] Y. C. Eldar and M. Unser, "Nonideal sampling and interpolation from noisy observations in shift-invariant spaces," IEEE Trans. Signal Process., vol. 54, no. 7, pp. 2636-2651, Jul. 2006.

[21] S. Ramani, D. Van De Ville, T. Blu, and M. Unser, "Nonideal sampling and regularization theory," IEEE Trans. Signal Process., vol. 56, no. 3, pp. 1055-1070, 2008.

[22] T. Michaeli and Y.C. Eldar, "High rate interpolation of random signals from nonideal samples," IEEE Trans. Signal Process., vol. 57, no. 3, 2009.

[23] S. Ramani, D. Van De Ville, and M. Unser, "Non-ideal sampling and adapted reconstruction using the stochastic Matern model," in Proc. Int. Conf. Acoust., Speech, Signal Process. (ICASSP'06), 2006, vol. 2.

[24] M. Bownik, "The structure of shift-modulation invariant spaces: The rational case," J. Funct. Anal., vol. 244, no. 1, pp. 172-219, 2007.

[25] Y. C. Eldar, E. Matusiak, and T. Werther, "A constructive inversion framework for twisted convolution," Monatsh. Math., vol. 150, no. 4, 2007.

[26] K. Gröchenig, Foundations of Time-Frequency Analysis. Boston, MA: Birkhäuser, 2001.

[27] A. Ron and Z. Shen, "Weyl-Heisenberg frames and Riesz bases in $L_{2}\left(\mathbb{R}^{d}\right)$," Duke Math. J., vol. 89, no. 2, pp. 237-282, 1997.

[28] T. Werther, Y. C. Eldar, and N. K. Subbana, "Dual Gabor frames: Theory and computational aspects," IEEE Trans. Signal Process., vol. 53, no. 11, pp. 4147-4158, 2005

[29] T. Werther, E. Matusiak, Y. C. Eldar, and N. K. Subbana, "A unified approach to dual Gabor windows," IEEE Trans. Signal Process., vol. 55, no. 5, pp. 1758-1768, May 2007.

[30] T. Michaeli and Y. C. Eldar, "Optimization techniques in modern sampling theory," in Convex Optimization in Signal Processing and Communications, Y. C. Eldar and D. Palomar, Eds. Cambridge, U.K.: Cambridge Univ. Press, 2010.

[31] Y. C. Eldar and T. Werther, "General framework for consistent sampling in hilbert spaces," Int. J. Wavelets, Multiresolution, and Inf. Process., vol. 3, no. 3, pp. 347-359, Sep. 2005.

[32] A. Aldroubi, "Oblique projections in atomic spaces," Proc. Amer. Math. Soc., vol. 124, no. 7, pp. 2051-2060, 1996.

[33] Y. C. Eldar, "Sampling and reconstruction in arbitrary spaces and oblique dual frame vectors," J. Fourier Analys. Appl., vol. 1, no. 9, pp. 77-96, Jan. 2003.

[34] Y. C. Eldar, "Sampling without input constraints: Consistent reconstruction in arbitrary spaces," in Sampling, Wavelets and Tomogr., A. I. Zayed and J. J. Benedetto, Eds. Boston, MA: Birkhäuser, 2004, pp. 33-60.

[35] Y. C. Eldar, A. Ben-Tal, and A. Nemirovski, "Linear minimax regret estimation of deterministic parameters with bounded data uncertainties," IEEE Trans. Signal Process., vol. 52, pp. 2177-2188, Aug. 2004.

[36] Y. C. Eldar and N. Merhav, "A competitive minimax approach to robust estimation of random parameters," IEEE Trans. Signal Process., vol. 52, pp. 1931-1946, 2004.

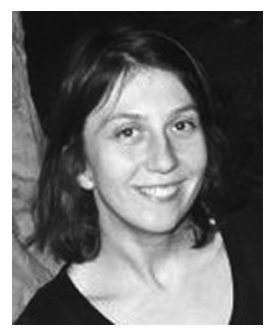

Ewa Matusiak received the Master's degree in mathematics and electrical engineering from Oklahoma University, Norman, in 2003, and the Ph.D. degree in mathematics from the University of Vienna, Austria, in 2007.

Currently she is a Postdoctoral Fellow with the Department of Electrical Engineering, Technion-Israel Institute of Technology, Haifa. She was a researcher with the Faculty of Mathematics, University of Vienna, from 2007 to 2008. In 2002, she was awarded a Richard V. Andree Memorial Scholarship and from 2003 to 2006, she held a Bertha von Suttner scholarship. In 2009, she was awarded the Andrew and Erna Finci Viterbi Fellowship. Her interests lie in Gabor analysis, sampling theory, uncertainty principle, and wireless communication. 


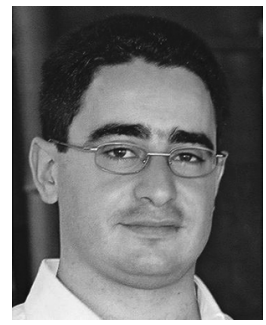

Tomer Michaeli (S'09) received the B.Sc. degree in electrical engineering in 2004 (summa cum laude) from the Technion-Israel Institute of Technology, Haifa.

$\mathrm{He}$ is currently pursuing the $\mathrm{Ph} . \mathrm{D}$. degree in electrical engineering at the Technion. From 2000 to 2008, he was a Research Engineer with RAFAEL Research Laboratories, Israel Ministry of Defense, Haifa. In 2008, he held the Andrew and Erna Finci Viterbi Fellowship. His research interests include statistical signal processing, estimation theory, sampling theory, image processing, and computer vision.

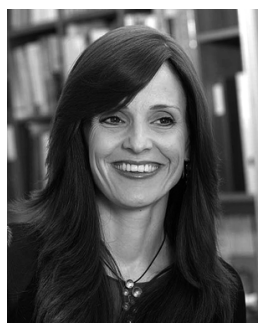

Yonina C. Eldar (S'97-M'02-SM'07) received the B.Sc. degree in physics in 1995 and the B.Sc. degree in electrical engineering in 1996, both from Tel-Aviv University (TAU), Tel-Aviv, Israel, and the Ph.D. degree in electrical engineering and computer science in 2001 from the Massachusetts Institute of Technology (MIT), Cambridge.

From January 2002 to July 2002, she was a Postdoctoral Fellow with the Digital Signal Processing Group, MIT. She is currently an Associate Professor with the Department of Electrical Engineering, Technion-Israel Institute of Technology, Haifa. She is also a Research Affiliate with the Research Laboratory of Electronics, MIT.

Dr. Eldar was in the Program For Outstanding Students at TAU from 1992 to 1996. In 1998, she held the Rosenblith Fellowship for study in Electrical Engineering at MIT, and in 2000, she held an IBM Research Fellowship. From 2002 to 2005, she was a Horev Fellow of the Leaders in Science and Technology program at the Technion and an Alon Fellow. In 2004, she was awarded the Wolf Foundation Krill Prize for Excellence in Scientific Research, in 2005 the Andre and Bella Meyer Lectureship, in 2007 the Henry Taub Prize for Excellence in Research, in 2008 the Hershel Rich Innovation Award, the Award for Women with Distinguished Contributions, the Muriel and David Jacknow Award for Excellence in Teaching, and the Technion Outstanding Lecture Award, and in 2009 the Technion's Award for Excellence in Teaching. She is a member of the IEEE Signal Processing Theory and Methods Technical Committee and the Bio Imaging Signal Processing Technical Committee, an Associate Editor for the IEEE TRANSACTIONS ON SIGNAL PROCESSING, the EURASIP Journal of Signal Processing, the SIAM Journal on Matrix Analysis and Applications, and the SIAM Journal on Imaging Sciences, and on the Editorial Board of Foundations and Trends in Signal Processing. 